\title{
Doubly nonlinear parabolic equations for a general class of Forchheimer gas flows in porous media
}

\author{
Emine Celik ${ }^{\dagger}$, Luan Hoang ${ }^{\dagger}$, and Thinh Kieu ${ }^{\ddagger}$ \\ March 22, 2022 \\ ${ }^{\dagger}$ Department of Mathematics and Statistics, Texas Tech University, Box 41042, Lubbock, TX \\ 79409-1042, U. S. A. \\ ${ }^{\ddagger}$ Department of Mathematics, University of North Georgia, Gainesville Campus, 3820 Mundy Mill \\ Rd., Oakwood, GA 30566, U. S. A. \\ Email addresses: emine.celik@ttu.edu, luan.hoang@ttu.edu, thinh.kieu@ung.edu
}

\begin{abstract}
This paper is focused on the generalized Forchheimer flows of compressible fluids in porous media. The gravity effect and other general nonlinear forms of the source terms and boundary fluxes are integrated into the model. It covers isentropic gas flows, ideal gases and slightly compressible fluids. We derive a doubly nonlinear parabolic equation for the so-called pseudopressure, and study the corresponding initial boundary value problem. The maximum estimates of the solution are established by using suitable trace theorem and adapting appropriately the Moser's iteration. The gradient estimates are obtained under a theoretical condition which, indeed, is relevant to the fluid flows in applications.
\end{abstract}

\section{Introduction}

We consider fluid flows in porous media with pressure $p$, density $\rho$, velocity $v$, and absolute viscosity $\mu$. The media has permeability $k>0$ and porosity $\phi \in(0,1)$. For hydrodynamics in porous media, the following Darcy's equation is usually used as a default law

$$
-\nabla p=\frac{\mu}{k} v
$$

However, even Darcy himself [5] noted that there were deviations from the linear equation (1.1). For instance, in the case the Reynolds number is large or fluids in fractured media, (1.1) becomes inaccurate in describing the fluid dynamics. Many work have been devoted to developing alternative nonlinear models to Darcy's law, see e.g. [3]. Forchheimer, in [9, 10], established the following three models:

$$
\begin{gathered}
-\nabla p=a v+b|v| v \\
-\nabla p=a v+b|v| v+c|v|^{2} v \\
-\nabla p=a v+d|v|^{m-1} v, \quad \text { for some real number } m \in(1,2) .
\end{gathered}
$$

The numbers $a, b, c, d$ above are empirical positive constants. The equations (1.2), (1.3) and (1.4) are usually referred to as Forchheimer's two-term, three-term and power laws, respectively. For more models and discussions, see [3, 25, 26, 28, 33] and references therein. 
From the mathematical point of view, the Darcy flows, under the umbrella "porous medium equations", have been analyzed intensively since 1960s, see e.g. [31] and a large number of references cited there. In contrast, the mathematics of Forchheimer flows and their variations has attracted much less attention. Moreover, the existing papers on this topic mainly treat incompressible fluids, leaving compressible ones barely studied, see [28] and references therein. The current paper aims to explore Forchheimer flows of compressible fluids using analytical techniques from partial differential equation (PDE) theory.

The Forchheimer equations (1.2), (1.3), (1.4) are extended to more general form

$$
-\nabla p=\sum_{i=0}^{N} a_{i}|v|^{\alpha_{i}} v,
$$

where $a_{i}$ 's are positive constants. Equation (1.5) is called the generalized Forchheimer equation. It is used to unify the models (1.2), (1.3), (1.4), and as a framework for interpretation of different experimental or field data. It is analyzed numerically in [8,21,27], theoretically in [2,12, 15, 18, for single-phase flows, and also in [16, 17] for two-phase flows.

For compressible fluids, especially gases, the dependence of coefficients $a_{i}$ 's on the density $\rho$ is essential. By using dimension analysis, Muskat [25] and then Ward [33] proposed the following equation for both laminar and turbulent flows in porous media:

$$
-\nabla p=f\left(v^{\alpha} k^{\frac{\alpha-3}{2}} \rho^{\alpha-1} \mu^{2-\alpha}\right), \text { where } f \text { is a function of one variable. }
$$

Using this, Ward [33] established from experimental data that

$$
-\nabla p=\frac{\mu}{k} v+c_{F} \frac{\rho}{\sqrt{k}}|v| v \quad \text { with } c_{F}>0 .
$$

This model is widely accepted as the standard form of the Forchheimer's two term-law.

Based on the arguments by Muskat and Ward, we proposed in [4] the following adaptation for (1.5)

$$
-\nabla p=\sum_{i=0}^{N} a_{i} \rho^{\alpha_{i}}|v|^{\alpha_{i}} v,
$$

where $N \geq 1, \alpha_{0}=0<\alpha_{1}<\ldots<\alpha_{N}$ are real numbers, the coefficients $a_{0}, \ldots, a_{N}$ are positive. This equation covers the two-term case (1.7), and the focus is the dependence on the density, but not viscosity and permeability.

Equation (1.8), however, does not take into account the gravity. Because of the nonlinear density-dependence of the model, any addition of new density terms may complicate the analysis as we will see below. Nonetheless, the gravity can be integrated into (1.8) by replacing $(-\nabla p)$ with $-\nabla p+\rho \vec{g}$, where $\vec{g}$ is the constant gravitational field. Therefore, we consider

$$
\sum_{i=0}^{N} a_{i} \rho^{\alpha_{i}}|v|^{\alpha_{i}} v=-\nabla p+\rho \vec{g} .
$$

Denote by $g: \mathbb{R}^{+} \rightarrow \mathbb{R}^{+}$a generalized polynomial with positive coefficients defined by

$$
g(s)=a_{0} s^{\alpha_{0}}+a_{1} s^{\alpha_{1}}+\cdots+a_{N} s^{\alpha_{N}} \quad \text { for } s \geq 0,
$$

with $a_{0}, a_{1}, \ldots, a_{N}>0$. Then (1.9) can be rewritten as

$$
g(\rho|v|) v=-\nabla p+\rho \vec{g}
$$


With this rule, we now follow the method in [4] to derive the basic PDE and its corresponding boundary condition. Multiplying both sides of (1.11) by $\rho$, we obtain

$$
g(|\rho v|) \rho v=-\rho \nabla p+\rho^{2} \vec{g} .
$$

Solving for $\rho v$ from (1.12) gives

$$
\rho v=-K\left(\left|\rho \nabla p-\rho^{2} \vec{g}\right|\right)\left(\rho \nabla p-\rho^{2} \vec{g}\right),
$$

where the function $K: \mathbb{R}^{+} \rightarrow \mathbb{R}^{+}$is defined for $\xi \geq 0$ by

$$
K(\xi)=\frac{1}{g(s(\xi))},
$$

with $s(\xi)=s$ being the unique non-negative solution of $s g(s)=\xi$.

Relation (1.13) will be combined with other equations of fluid mechanics. The first is the continuity equation

$$
\phi \rho_{t}+\operatorname{div}(\rho v)=F
$$

where the porosity $\phi$ is a constant in $(0,1)$, the source term $F$ counts for the rate of net mass production or loss due to any source and/or sink in the media.

From (1.13) and (1.15) follows

$$
\phi \rho_{t}=\operatorname{div}\left(K\left(\left|\rho \nabla p-\rho^{2} \vec{g}\right|\right)\left(\rho \nabla p-\rho^{2} \vec{g}\right)\right)+F .
$$

We consider below scenarios of isentropic gas flows, ideal gases and slightly compressible fluids.

Isentropic gas flows. In this case

$$
p=\bar{c} \rho^{\gamma} \quad \text { for some constants } \bar{c}, \gamma>0 .
$$

Here, $\gamma$ is the specific heat ratio. Note that $\rho \nabla p=\nabla\left(\bar{c} \gamma \rho^{\gamma+1} /(\gamma+1)\right)$. Hence by letting

$$
u=\frac{\bar{c} \gamma \rho^{\gamma+1}}{\gamma+1}=\frac{\gamma p^{\frac{\gamma+1}{\gamma}}}{\bar{c}^{\frac{1}{\gamma}}(\gamma+1)}
$$

we rewrite (1.16) as

$$
\phi c^{1 / 2}\left(u^{\lambda}\right)_{t}=\nabla \cdot\left(K\left(\left|\nabla u-c u^{\ell} \vec{g}\right|\right)\left(\nabla u-c u^{\ell} \vec{g}\right)\right)+F
$$

where

$$
\lambda=\frac{1}{\gamma+1} \in(0,1), \quad \ell=2 \lambda \quad \text { and } \quad c=\left(\frac{\gamma+1}{\bar{c} \gamma}\right)^{\ell} .
$$

The new quantity $u$ in (1.18) is essentially a pseudo-pressure.

Ideal gases. The equation of state is

$$
p=\bar{c} \rho \quad \text { for some constant } \bar{c}>0 \text {. }
$$

We can consider (1.21) as a special case of (1.17) with $\gamma=1$, and derive the same equation (1.19) with $\lambda=1 / 2$. In this case, the pseudo-pressure $u \sim p^{2}$ which is used commonly in engineering problems.

Although we mainly focus on gases, we also present here, in a unified way, the slightly compressible fluids which is important in petroleum engineering. 
Slightly compressible fluids. The equation of state is

$$
\frac{1}{\rho} \frac{d \rho}{d p}=\frac{1}{\kappa}=\text { const. }>0 \text {. }
$$

Then $\rho \nabla p=\kappa \nabla \rho$, and by letting

$$
u=\kappa \rho, \quad \ell=2 \quad \text { and } \quad c=1 / \kappa^{2},
$$

we obtain the same equation (1.19) with $\lambda=1$.

For all three cases, by scaling the time variable, we can always assume the multiplying factor on the left-hand side of (1.19) to be 1 . In summary, we have derived

$$
\left(u^{\lambda}\right)_{t}=\nabla \cdot\left(K\left(\left|\nabla u-c u^{\ell} \vec{g}\right|\right)\left(\nabla u-c u^{\ell} \vec{g}\right)\right)+F
$$

with constants $\lambda \in(0,1], \ell=2 \lambda, c>0$, and function $F(x, t)$ being rescaled appropriately.

Boundary condition. We will study the problem in a bounded domain $U$ with outward normal vector $\vec{\nu}$ on the boundary. We consider the volumetric flux condition

$$
v \cdot \vec{\nu}=\psi \text { on } \partial U
$$

This gives $\rho v \cdot \vec{\nu}=\psi \rho$, hence, together with (1.13) and (1.18) or (1.22) yields

$$
-K\left(\left|\nabla u+c u^{\ell} \vec{g}\right|\right)\left(\nabla u+c u^{\ell} \vec{g}\right) \cdot \vec{\nu}=c^{1 / 2} \psi u^{\lambda} .
$$

General formulation and the initial boundary value problem (IBVP). Although problem (1.23) and (1.24) is our motivation, in this mathematical investigation, we consider a more general class of equations and boundary conditions, namely,

$$
\begin{cases}\frac{\partial\left(u^{\lambda}\right)}{\partial t}=\nabla \cdot(K(|\nabla u+Z(u)|)(\nabla u+Z(u)))+f(x, t, u) & \text { on } U \times(0, \infty), \\ u(x, 0)=u_{0}(x) & \text { on } U, \\ K(|\nabla u+Z(u)|)(\nabla u+Z(u)) \cdot \vec{\nu}=B(x, t, u) & \text { on } \Gamma \times(0, \infty),\end{cases}
$$

where $Z(u)$ is a function from $[0, \infty)$ to $\mathbb{R}^{n}, B(x, t, u)$ is a function from $\Gamma \times[0, \infty) \times[0, \infty)$ to $\mathbb{R}$ and $f(x, t, u)$ is a function from $U \times[0, \infty) \times[0, \infty)$ to $\mathbb{R}$.

In (1.25) the source term $f(x, t, u)$ now can depend on $u$, and the boundary term $B(x, t, u)$ can be more general than $\psi(x, t) u^{\lambda}$. For our analysis, they still need some growth conditions.

Assumption (A1). Throughout this paper, we assume that functions $Z(u):[0, \infty) \rightarrow \mathbb{R}^{n}$, $B(x, t, u): \Gamma \times[0, \infty) \times[0, \infty) \rightarrow \mathbb{R}$ and $f(x, t, u): U \times[0, \infty) \times[0, \infty) \rightarrow \mathbb{R}$ satisfy

$$
\begin{gathered}
|Z(u)| \leq d_{0} u^{\ell_{Z}}, \\
B(x, t, u) \leq \varphi_{1}(x, t)+\varphi_{2}(x, t) u^{\ell_{B}}, \\
f(x, t, u) \leq f_{1}(x, t)+f_{2}(x, t) u^{\ell_{f}}
\end{gathered}
$$

with constants $d_{0}, \ell_{Z}>0, \ell_{f}, \ell_{B} \geq 0$, and functions $\varphi_{1}, \varphi_{2}, f_{1}, f_{2} \geq 0$.

Note that the growths with respect to $u$ in Assumption (A1) are arbitrary. This is different from other existing papers when the exponents $\ell_{Z}, \ell_{f}, \ell_{B}$ are restricted to suit certain Sobolev embedding or trace theorems. 
The problem (1.25) is significantly more general than the one in our previous work [4]. It can arise from other complex, nonlinear models of fluid flows in porous media. Indeed, a similar PDE with $p$-Laplacian structure was derived in [7] for water vapor. Here, we showed that it comes naturally from the Forchheimer equations and is formulated for the pseudo-pressure $u$ instead. Due to more complicated flows (1.11), the resulting function $K(\xi)$ is non-homogeneous compared to the homogeneous one in [7].

Regarding the PDE in (1.25), it is doubly nonlinear in both $u$ and $\nabla u$. (For the theory of equations of this type, see the monograph [19], review paper [20] and e.g. [1,22, 23, 30,32].) Moreover, our equation contains lower order terms of arbitrary growths in $u$. Therefore, it is not clear whether $L^{\infty}$-estimates are possible. In addition, the boundary condition is time-dependent, of non-linear Robin type and also has arbitrary growth rate in $u$. Thus, the boundary contribution is not trivial and, thanks to the nonlinearity, we cannot shift the solution by subtracting the boundary data. For vanishing Dirichlet boundary condition, the work [7] uses maximum principle which is not applicable to our problem. Also, both [7,30] imposes the $L^{\infty}$-requirement for the initial data. In contrast, we use Moser's iteration [24, hence can deal with more complex equation and boundary condition. Furthermore, we only require the initial data belonging to a certain $L^{\alpha}$-space with finite $\alpha>0$, and derive the $L^{\infty}$-estimates of the solution for positive time. We note that, although ours are a priori estimates, they are crucial in establishing, via regularization and approximation, the existence results (see [19]).

The paper is organized as follows. In section 2, we recall needed trace theorem and PoincaréSobolev inequalities. In particular, the inequalities in Corollary 2.2 are formulated to suit the nonlinear diffusion, and later treatment of the general nonlinearity in the source term and Robin boundary condition. In section 3 , we establish the $L^{\alpha}$-estimate of a solution $u(x, t)$ of (1.25) for any finite $\alpha>0$ in terms of the initial and boundary data. In section 4 , we derive an estimate for spatially global $L^{\infty}$-norm of $u(x, t)$ in Theorem 4.6 by adapting Moser's iteration. The sequence of exponents in the iteration are constructed based on the nonlinearity of the boundary condition and the source term. We note that the global $L^{\infty}$-norm of $u$ is bounded by (4.38) in Theorem 4.4 which is "quasi-homogeneous" in its $L_{x, t}^{\beta}$-norm for some $\beta>0$. This extends previous results in [4, 29]. In section [5, we establish $L^{2-a}$-estimates for the gradient of $u(x, t)$ in Theorem [5.2, Even for this simple norm, it is non-trivial due to the arbitrary growth in the nonlinear Robin boundary condition. It is obtained under condition (5.8). This mathematical requirement turns out to be naturally satisfied for the original problem (1.23) and known gases such as those in the data book [34]. It modestly shows the relevance of our mathematical analysis.

\section{Basic inequalities}

First, we recall elementary inequalities that will be used frequently. Let $x, y \geq 0$. Then

$$
\begin{aligned}
& (x+y)^{p} \leq x^{p}+y^{p} \quad \text { for all } 0<p \leq 1, \\
& (x+y)^{p} \leq 2^{p-1}\left(x^{p}+y^{p}\right) \quad \text { for all } p \geq 1, \\
& x^{\beta} \leq x^{\alpha}+x^{\gamma} \quad \text { for all } 0 \leq \alpha \leq \beta \leq \gamma,
\end{aligned}
$$

particularly,

$$
x^{\beta} \leq 1+x^{\gamma} \text { for all } 0 \leq \beta \leq \gamma .
$$

Next, we recall particular Poincáre-Sobolev inequality and trace theorem. 
The classical trace theorem: If a function $u(x)$ belongs to $W^{1,1}(U)$ then

$$
\int_{\Gamma}|u| d \sigma \leq c_{1} \int_{U}|u| d x+c_{2} \int_{U}|\nabla u| d x
$$

where constants $c_{1}, c_{2}>0$ depend on $U$.

Lemma 2.1 ( [4, Lemma 2.1). In the following statements, $u(x)$ is a function defined on $U$.

(i) If $\alpha \geq s \geq 0, \alpha \geq 1$, and $p>1$, then for any $|u|^{\alpha} \in W^{1,1}(U)$ and $\varepsilon>0$ one has

$$
\int_{\Gamma}|u|^{\alpha} d \sigma \leq \varepsilon \int_{U}|u|^{\alpha-s}|\nabla u|^{p} d x+c_{1} \int_{U}|u|^{\alpha} d x+\left(c_{2} \alpha\right)^{\frac{p}{p-1}} \varepsilon^{-\frac{1}{p-1}} \int_{U}|u|^{\alpha+\frac{s-p}{p-1}} d x .
$$

(ii) If $n>p>1, r>0, \alpha \geq s \geq 0, \alpha \geq \frac{p-s}{p-1}$, and $\alpha>\frac{n(r+s-p)}{p}$, then for any $\varepsilon>0$ one has

$$
\begin{aligned}
\int_{U}|u|^{\alpha+r} d x \leq \varepsilon \int_{U}|u|^{\alpha-s}|\nabla u|^{p} d x+\varepsilon^{-\frac{\theta}{1-\theta}} 2^{\frac{\theta(\alpha-s+p)}{1-\theta}}\left(\bar{c}_{3} m\right)^{\frac{\theta p}{1-\theta}}\|u\|_{L^{\alpha}}^{\alpha+\mu} \\
+2^{\theta(\alpha-s+p)} \frac{\theta p}{c_{4}}|U|^{\frac{\theta(\alpha(p-1)+s-p)}{\alpha}}\|u\|_{L^{\alpha}}^{\alpha+r},
\end{aligned}
$$

for all $|u|^{m} \in W^{1, p}(U)$, where

$$
m=\frac{\alpha-s+p}{p}, \quad \theta=\frac{r}{(\alpha p / n)+p-s}, \quad \mu=\frac{r+\theta(s-p)}{1-\theta}
$$

and positive constants $\bar{c}_{3}=\bar{c}_{3}(U, p)$ and $\bar{c}_{4}=\bar{c}_{4}(U, p)$ depend on $U, p$, but not on $u(x), \alpha, s$.

The following remarks on Lemma 2.1 are in order.

(a) We can calculate $r$ in terms of $\theta$ by the second formula in (2.8), and rewrite the power $\alpha+\mu$ in (2.7) as

$$
\alpha+\mu=\alpha+\left[\theta\left(\frac{\alpha p}{n}+(p-s)\right)+\theta(s-p)\right] \frac{1}{1-\theta}=\alpha\left(1+\frac{p}{n} \cdot \frac{\theta}{1-\theta}\right) .
$$

Assume $p \leq s$, then $m \leq \alpha$ and we can rewrite (2.7) as

$$
\int_{U}|u|^{\alpha+r} d x \leq \varepsilon \int_{U}|u|^{\alpha-s}|\nabla u|^{p} d x+\bar{D}_{1}\|u\|_{L^{\alpha}}^{\alpha+r}+\varepsilon^{-\frac{\theta}{1-\theta}} \bar{D}_{2}\|u\|_{L^{\alpha}}^{\alpha\left(1+\frac{p}{n} \cdot \frac{\theta}{1-\theta}\right)},
$$

where

$$
\bar{D}_{1}=\left[2^{\alpha-s+p} \bar{c}_{4}^{p}|U|^{\frac{\alpha(p-1)+s-p}{\alpha}}\right]^{\theta}, \quad \bar{D}_{2}=\left[2^{\alpha-s+p}\left(\bar{c}_{3} \alpha\right)^{p}\right]^{\frac{\theta}{1-\theta}} .
$$

(b) For any $r \geq 0, \alpha \geq s \geq 0, \alpha+r \geq 1$, applying (2.6) for $\alpha \rightarrow \alpha+r$ and $s \rightarrow s+r$ yields

$$
\int_{\Gamma}|u|^{\alpha+r} d \sigma \leq \varepsilon \int_{U}|u|^{\alpha-s}|\nabla u|^{p} d x+c_{1} \int_{U}|u|^{\alpha+r} d x+\left(c_{2}(\alpha+r)\right)^{\frac{p}{p-1}} \varepsilon^{-\frac{1}{p-1}} \int_{U}|u|^{\alpha+\hat{r}} d x
$$

where

$$
\hat{r}=r+\frac{r+s-p}{p-1}=\frac{p r+s-p}{p-1} .
$$

In our particular case, we have following corollary.

Corollary 2.2. Assume $1>a>\delta \geq 0, \alpha \geq 2-\delta$, and $|u|^{\alpha} \in W^{1,1}(U)$. Let $r>0$. 
(i) For any $\varepsilon>0$ one has

$$
\begin{aligned}
\int_{\Gamma}|u|^{\alpha+r} d \sigma \leq \varepsilon \int_{U}|u|^{\alpha-2+\delta}|\nabla u|^{2-a} d x & +c_{1} \int_{U}|u|^{\alpha+r} d x \\
& +\left(c_{2}(\alpha+r)\right)^{\frac{2-a}{1-a}} \varepsilon^{-\frac{1}{1-a}} \int_{U}|u|^{\alpha+\frac{(2-a) r+a-\delta}{1-a}} d x
\end{aligned}
$$

(ii) If $\alpha>\frac{n(r+a-\delta)}{2-a}$, then for any $\varepsilon>0$ one has

$$
\int_{U}|u|^{\alpha+r} d x \leq \varepsilon \int_{U}|u|^{\alpha-2+\delta}|\nabla u|^{2-a} d x+D_{1}\|u\|_{L^{\alpha}}^{\alpha+r}+D_{2} \varepsilon^{-\frac{\theta}{1-\theta}}\|u\|_{L^{\alpha}}^{\alpha\left(1+\frac{2-a}{n} \cdot \frac{\theta}{1-\theta}\right)},
$$

where

$$
\begin{aligned}
\theta & =\frac{r}{\alpha(2-a) / n+\delta-a}, \\
D_{1} & =\left(2^{\alpha+\delta-a} c_{4}^{2-a}|U|^{\frac{\alpha(1-a)+a-\delta}{\alpha}}\right)^{\theta}, \quad D_{2}=\left(2^{\alpha+\delta-a}\left(c_{3} \alpha\right)^{2-a}\right)^{\frac{\theta}{1-\theta}}
\end{aligned}
$$

with $c_{3}=\bar{c}_{3}(U, 2-a)>0$ and $c_{4}=\bar{c}_{4}(U, 2-a)>0$.

Proof. Let $p=2-a$ and $s=2-\delta$, noticing that $p<s$. Then inequality (2.12) follows (2.11), and inequality (2.13) follows (2.10).

We also recall a particular multiplicative parabolic Sobolev inequality.

Lemma 2.3 ( 4], Lemma 2.3). Assume $1>a>\delta \geq 0$,

$$
\alpha \geq 2-\delta \quad \text { and } \quad \alpha>\alpha_{*} \stackrel{\text { def }}{=} \frac{n(a-\delta)}{2-a} .
$$

If $T>0$, then

$$
\begin{aligned}
\left(\int_{0}^{T} \int_{U}|u|^{\kappa \alpha} d x d t\right)^{\frac{1}{\kappa \alpha}} \leq & \left(c_{5} \alpha^{2-a}\right)^{\frac{1}{\kappa \alpha}}\left(\int_{0}^{T} \int_{U}|u|^{\alpha+\delta-a} d x d t+\int_{0}^{T} \int_{U}|u|^{\alpha+\delta-2}|\nabla u|^{2-a} d x d t\right)^{\frac{\tilde{\theta}}{\alpha+\delta-a}} \\
& \cdot \sup _{t \in[0, T]}\left(\int_{U}|u(x, t)|^{\alpha} d x\right)^{\frac{1-\tilde{\theta}}{\alpha}}
\end{aligned}
$$

where $c_{5} \geq 1$ is independent of $\alpha$ and $T$, and

$$
\tilde{\theta}=\tilde{\theta}_{\alpha} \stackrel{\text { def }}{=} \frac{1}{1+\frac{\alpha(2-a)}{n(\alpha+\delta-a)}}, \quad \kappa=\kappa(\alpha) \stackrel{\text { def }}{=} 1+\frac{2-a}{n}-\frac{a-\delta}{\alpha}=1+(a-\delta)\left(\frac{1}{\alpha_{*}}-\frac{1}{\alpha}\right) .
$$

\section{Estimates of the Lebesgue norms}

From now on, we fix a function $g(s)$ in (1.10) and (1.11). Denote

$$
a=\frac{\alpha_{N}}{\alpha_{N}+1} \in(0,1) .
$$

This number will be used in our calculations throughout. 
The function $K(\xi)$ defined by (1.14) has the following properties: it is decreasing in $\xi$, maps $[0, \infty)$ onto $\left(0, \frac{1}{a_{0}}\right]$ and

$$
\begin{array}{r}
\frac{d_{1}}{(1+\xi)^{a}} \leq K(\xi) \leq \frac{d_{2}}{(1+\xi)^{a}}, \\
d_{3}\left(\xi^{2-a}-1\right) \leq K(\xi) \xi^{2} \leq d_{2} \xi^{2-a},
\end{array}
$$

where $d_{1}, d_{2}, d_{3}$ are positive constants depending on $\alpha_{i}$ 's and $a_{i}$ 's of the function $g(s)$, see [2]. Let $n=2,3, \ldots$, and $U$ be the a bounded, open, connected subset of $\mathbb{R}^{n}$ with $C^{2}$ boundary $\Gamma=\partial U$. In applications, $n=2$ or 3 , but we treat all $n \geq 2$ in this paper.

Hereafter, $u(x, t) \geq 0$ is a solution of IBVP (1.25).

Define $\delta=1-\lambda \in[0,1)$. Throughout the paper, we assume

$$
a>\delta .
$$

This assumption is to avoid too many possible cases for our estimates. The case $a \leq \delta$ can be treated similarly and, in fact, is easier to deal with.

Then $\alpha_{*}=n(a-\delta) /(2-a)$ in (2.14) is a fixed positive number. In order to describe our results, we introduce some constants and quantities. Define

$$
\kappa_{B}=1+\frac{1-a}{n} \text { and } \quad \kappa_{f}=1+\frac{2-a}{n} .
$$

Let $p_{1}, p_{2}, p_{3}, p_{4}$ be fixed numbers such that

$$
1<p_{1}, p_{2}<\kappa_{B} \text { and } 1<p_{3}, p_{4}<\kappa_{f} .
$$

For $i=1,2,3,4$ let $q_{i}$ be the conjugate exponent of $p_{i}$, that is, $1 / p_{i}+1 / q_{i}=1$. Then (3.6) is equivalent to

$$
1+\frac{n}{1-a}<q_{1}, q_{2}<\infty \text { and } 1+\frac{n}{2-a}<q_{3}, q_{4}<\infty .
$$

A key quantity in expressing our estimates is

$$
\Upsilon(t)=\left\|\varphi_{1}(t)\right\|_{L^{q_{1}}(\Gamma)}^{q_{1}}+\left\|\varphi_{2}(t)\right\|_{L^{q_{2}(\Gamma)}}^{q_{2}}+\left\|f_{1}(t)\right\|_{L^{q_{3}(U)}}^{q_{3}}+\left\|f_{2}(t)\right\|_{L^{q_{4}}(U)}^{q_{4}} \quad \text { for } t \geq 0 .
$$

We also denote by $\eta_{0}$ a positive number defined by

$$
\begin{aligned}
\eta_{0}=\max \{ & q_{1} \lambda, q_{2}\left(\lambda-\ell_{B}\right), n\left(\ell_{Z}-1\right), \frac{-p_{1} \lambda+a-\delta}{\kappa_{f}-p_{1}}, \frac{p_{2}\left(-\lambda+\ell_{B}\right)+a-\delta}{\kappa_{f}-p_{2}}, \\
& \left.\frac{-p_{1} \lambda+(a-\delta)}{\kappa_{B}-p_{1}}, \frac{p_{2}\left(-\lambda+\ell_{B}\right)+a-\delta}{\kappa_{B}-p_{2}}, \frac{-p_{3} \lambda+a-\delta}{\kappa_{f}-p_{3}}, \frac{p_{4}\left(-\lambda+\ell_{f}\right)+a-\delta}{\kappa_{f}-p_{4}}\right\} .
\end{aligned}
$$

In the following, we focus on estimating the $L^{\alpha}$-norm on $U$ of the solution $u(x, t)$, for any given $\alpha>0$ and $t>0$. We start with a differential inequality for $\|u(t)\|_{L^{\alpha}(U)}^{\alpha}$.

Lemma 3.1. Assume

$$
\alpha \geq \max \left\{2, \frac{n a}{1-a}\right\} \quad \text { and } \quad \alpha>\eta_{0}
$$

For $t>0$, one has

$$
\frac{d}{d t} \int_{U} u(x, t)^{\alpha} d x+\int_{U}|\nabla u(x, t)|^{2-a} u(x, t)^{\alpha-\lambda-1} d x \leq C_{0} \cdot\left(\|u(t)\|_{L^{\alpha}(U)}^{\nu_{1}}+\|u(t)\|_{L^{\alpha}(U)}^{\nu_{2}}+\Upsilon(t)\right),
$$


where $C_{0}=C_{0}(\alpha)>0$,

$$
\nu_{1}=\nu_{1}(\alpha) \stackrel{\text { def }}{=} \alpha-\lambda-1 \quad \text { and } \quad \nu_{2}=\nu_{2}(\alpha) \stackrel{\text { def }}{=} \alpha\left(1+\frac{2-a}{n} \cdot \frac{\theta}{1-\theta}\right)
$$

with

$$
\theta=\theta_{\alpha} \stackrel{\text { def }}{=} \frac{\nu_{3}-\alpha}{\alpha(2-a) / n+\delta-a} \text { for } \nu_{3}=\nu_{3}(\alpha) \text { defined by 3.24 below. }
$$

Proof. Multiplying the first equation in (1.25) by $u^{\alpha-\lambda}$, integrating over domain $U$, and using integration by parts we have

$$
\begin{aligned}
\frac{\lambda}{\alpha} \frac{d}{d t} \int_{U} u^{\alpha} d x= & \int_{U} \nabla \cdot(K(|\nabla u+Z(u)|)(\nabla u+Z(u))) u^{\alpha-\lambda} d x+\int_{U} f u^{\alpha-\lambda} d x \\
= & -(\alpha-\lambda) \int_{U} K(|\nabla u+Z(u)|)(\nabla u+Z(u)) \cdot \nabla u\left(u^{\alpha-\lambda-1}\right) d x \\
& +\int_{\Gamma} K(|\nabla u+Z(u)|)(\nabla u+Z(u)) \cdot \vec{\nu} u^{\alpha-\lambda} d \sigma+\int_{U} f u^{\alpha-\lambda} d x .
\end{aligned}
$$

For the first integral on the right-hand side, we write

$$
(\nabla u+Z(u)) \cdot \nabla u=|\nabla u+Z(u)|^{2}-(\nabla u+Z(u)) \cdot Z(u) .
$$

For the boundary integral, we use the boundary condition in (1.25). These result in

$$
\begin{aligned}
& \frac{\lambda}{\alpha} \frac{d}{d t} \int_{U} u^{\alpha} d x=-(\alpha-\lambda) \int_{U} K(|\nabla u+Z(u)|)|\nabla u+Z(u)|^{2} u^{\alpha-\lambda-1} d x \\
& +(\alpha-\lambda) \int_{U} K(|\nabla u+Z(u)|)(\nabla u+Z(u)) \cdot Z(u) u^{\alpha-\lambda-1} d x+\int_{\Gamma} u^{\alpha-\lambda} B d \sigma+\int_{U} f u^{\alpha-\lambda} d x .
\end{aligned}
$$

Using relations (1.26), (3.2) and (3.3) for the first two integrals on the right-hand side, as well as (1.27), (1.28) for the last two, we have

$$
\begin{aligned}
\frac{\lambda}{\alpha} \frac{d}{d t} \int_{U} u^{\alpha} d x \leq & -(\alpha-\lambda) d_{3} \int_{U}\left(|\nabla u+Z(u)|^{2-a}-1\right) u^{\alpha-\lambda-1} d x \\
& +(\alpha-\lambda) d_{2} d_{0} \int_{U}(|\nabla u+Z(u)|+1)^{1-a} u^{\alpha-\lambda-1+\ell_{z}} d x \\
& +\int_{\Gamma} u^{\alpha-\lambda}\left(\varphi_{1}+\varphi_{2} u^{\ell_{B}}\right) d \sigma+\int_{U}\left(f_{1}+f_{2} u^{\ell_{f}}\right) u^{\alpha-\lambda} d x
\end{aligned}
$$

Using (2.1), we estimate

$$
(|\nabla u+Z(u)|+1)^{1-a} \leq|\nabla u+Z(u)|^{1-a}+1 .
$$

Hence, we obtain

$$
\frac{\lambda}{\alpha} \frac{d}{d t} \int_{U} u^{\alpha} d x \leq-(\alpha-\lambda) d_{3} I_{1}+(\alpha-\lambda) d_{3} I_{2}+(\alpha-\lambda) d_{2} d_{0} I_{3}+(\alpha-\lambda) d_{2} d_{0} I_{4}+I_{5}+I_{6} .
$$

where

$$
\begin{aligned}
I_{1} & =\int_{U}|\nabla u+Z(u)|^{2-a} u^{\alpha-\lambda-1} d x, & I_{2} & =\int_{U} u^{\alpha-\lambda-1} d x, \\
I_{3} & =\int_{U}|\nabla u+Z(u)|^{1-a} u^{\alpha-\lambda-1+\ell_{z}} d x, & I_{4} & =\int_{U} u^{\alpha-\lambda-1+\ell_{z}} d x, \\
I_{5} & =\int_{\Gamma}\left(u^{\alpha-\lambda} \varphi_{1}+u^{\alpha-\lambda+\ell_{B}} \varphi_{2}\right) d \sigma, & I_{6} & =\int_{U}\left(u^{\alpha-\lambda} f_{1}+u^{\alpha-\lambda+\ell_{f}} f_{2}\right) d x .
\end{aligned}
$$


In calculations below, the positive constants $\bar{C}, C, C_{\varepsilon}, C_{\varepsilon}^{\prime}$ are generic, with $\bar{C}$ independent of $\alpha$, while $C$ depending on $\alpha$, and $C_{\varepsilon}, C_{\varepsilon}^{\prime}$ depending on $\alpha$ and $\varepsilon$.

- Applying Young's inequality to the integrand of $I_{3}$ with powers $\frac{2-a}{1-a}$ and $2-a$ gives

$$
I_{3} \leq \frac{d_{3}}{2 d_{2} d_{0}} \int_{U}|\nabla u+Z(u)|^{2-a} u^{\alpha-\lambda-1} d x+\bar{C} \int_{U} u^{\ell_{Z}(2-a)+\alpha-\lambda-1} d x=\frac{d_{3}}{2 d_{2} d_{0}} I_{1}+\bar{C} I_{7},
$$

where

$$
I_{7}=\int_{U} u^{\mu_{1}} d x \quad \text { with } \mu_{1}=\ell_{Z}(2-a)+\alpha-\lambda-1 .
$$

Note also, by (2.3), that

$$
I_{4} \leq I_{2}+I_{7}
$$

Then

$$
\frac{\lambda}{\alpha} \frac{d}{d t} \int_{U} u^{\alpha} d x \leq-(\alpha-\lambda) \frac{d_{3}}{2} I_{1}+\bar{C} \alpha\left(I_{2}+I_{7}\right)+I_{5}+I_{6}
$$

- To estimate $I_{1}$ we use inequality

$|x|^{2-a} \leq 2^{1-a}\left(|x-y|^{2-a}+|y|^{2-a}\right)$ which gives $|x-y|^{2-a} \geq 2^{a-1}|x|^{2-a}-|y|^{2-a} \quad \forall x, y \in \mathbb{R}^{n}$.

Together with (1.26), it gives

$$
\begin{aligned}
I_{1} & \geq \int_{U}\left(2^{a-1}|\nabla u|^{2-a} u^{\alpha-\lambda-1}-|Z(u)|^{2-a} u^{\alpha-\lambda-1}\right) d x \\
& \geq 2^{a-1} \int_{U}|\nabla u|^{2-a} u^{\alpha-\lambda-1} d x-d_{0}^{2-a} \int_{U} u^{\alpha-\lambda-1+\ell_{Z}(2-a)} d x \\
& \geq \frac{1}{2} \int_{U}|\nabla u|^{2-a} u^{\alpha-\lambda-1} d x-d_{0}^{2-a} I_{7} .
\end{aligned}
$$

This and (3.14) imply

$$
\frac{\lambda}{\alpha} \frac{d}{d t} \int_{U} u^{\alpha} d x+(\alpha-\lambda) \frac{d_{3}}{4} \int_{U}|\nabla u|^{2-a} u^{\alpha-\lambda-1} d x \leq \bar{C} \alpha\left(I_{2}+I_{7}\right)+I_{5}+I_{6}
$$

- For $I_{5}$, using Young's inequality, we have

$$
I_{5} \leq \int_{\Gamma} u^{\mu_{2}} d \sigma+\int_{\Gamma} u^{\mu_{3}} d \sigma+\left\|\varphi_{1}\right\|_{L^{q_{1}(\Gamma)}}^{q_{1}}+\left\|\varphi_{2}\right\|_{L^{q_{2}(\Gamma)}}^{q_{2}},
$$

where

$$
\mu_{2}=p_{1}(\alpha-\lambda) \quad \text { and } \quad \mu_{3}=p_{2}\left(\alpha-\lambda+\ell_{B}\right) .
$$

For the first two integrals on the right-hand side, applying (2.12) to $\alpha+r=\mu_{2}$ and $\alpha+r=\mu_{3}$ gives

$$
\begin{aligned}
\int_{\Gamma} u^{\mu_{2}} d \sigma+\int_{\Gamma} u^{\mu_{3}} d \sigma \leq & \varepsilon \int_{U}|u|^{\alpha-\lambda-1}|\nabla u|^{2-a} d x \\
& +c_{1} \int_{U}\left(u^{\mu_{2}}+u^{\mu_{3}}\right) d x+C_{\varepsilon} \int_{U}\left(u^{\mu_{4}}+u^{\mu_{5}}\right) d x
\end{aligned}
$$

where $\varepsilon>0$ is arbitrary,

$$
\begin{aligned}
& \mu_{4}=\alpha+\frac{(2-a) r+a-\delta}{1-a}=\alpha+\frac{(2-a)\left(\left(p_{1}-1\right) \alpha-p_{1} \lambda\right)+a-\delta}{1-a}>\alpha, \\
& \mu_{5}=\alpha+\frac{(2-a) r+a-\delta}{1-a}=\alpha+\frac{(2-a)\left(\left(p_{2}-1\right) \alpha+p_{2}\left(-\lambda+\ell_{B}\right)\right)+a-\delta}{1-a}>\alpha .
\end{aligned}
$$


(The validity of (3.19) will be verified later.) Then

$$
I_{5} \leq \varepsilon \int_{U}|u|^{\alpha-\lambda-1}|\nabla u|^{2-a} d x+C_{\varepsilon} \int_{U} \sum_{i=2}^{5} u^{\mu_{i}} d x+\left\|\varphi_{1}\right\|_{L^{q_{1}(\Gamma)}}^{q_{1}}+\left\|\varphi_{2}\right\|_{L^{q_{2}}(\Gamma)}^{q_{2}} .
$$

- For $I_{6}$, using Young's inequalities we have

$$
I_{6} \leq \int_{U}\left(u^{\mu_{6}}+u^{\mu_{7}}\right) d x+\left\|f_{1}\right\|_{L^{q_{3}}}^{q_{3}}+\left\|f_{2}\right\|_{L^{q_{4}}}^{q_{4}},
$$

where

$$
\mu_{6}=p_{3}(\alpha-\lambda) \quad \text { and } \quad \mu_{7}=p_{4}\left(\alpha-\lambda+\ell_{f}\right) .
$$

Note for $1 \leq i \leq 7$ that $\nu_{1} \leq \mu_{i} \leq \nu_{3}$, where, referring to (3.13), (3.20) and (3.23),

$$
\nu_{3}=\max \left\{\mu_{i}: 1 \leq i \leq 7\right\} .
$$

Hence by using (2.3), $u^{\mu_{i}} \leq u^{\nu_{1}}+u^{\nu_{3}}$ for all $1 \leq i \leq 7$. Combining this with (3.16), (3.21), and (3.22) yields

$$
\frac{\lambda}{\alpha} \frac{d}{d t} \int_{U} u^{\alpha} d x+\left[(\alpha-\lambda) \frac{d_{3}}{4}-\varepsilon\right] \int_{U}|\nabla u|^{2-a} u^{\alpha-\lambda-1} d x \leq C_{\varepsilon} \int_{U}\left(u^{\nu_{1}}+u^{\nu_{3}}\right) d x+\Upsilon(t),
$$

Using Hölder's inequality we have

$$
\int_{U} u^{\nu_{1}} d x \leq C\left(\int_{U} u^{\alpha} d x\right)^{\frac{\nu_{1}}{\alpha}}=C\|u\|_{L^{\alpha}}^{\nu_{1}}
$$

Applying (2.13) to $\alpha+r=\nu_{3}$ gives

$$
C_{\varepsilon} \int_{U} u^{\nu_{3}} d x \leq \varepsilon \int_{U}|u|^{\alpha-\lambda-1}|\nabla u|^{2-a} d x+C_{\varepsilon}^{\prime}\|u\|_{L^{\alpha}}^{\nu_{3}}+C_{\varepsilon}^{\prime}\|u\|_{L^{\alpha}}^{\nu_{2}}
$$

(Again, we will verify the validity of (3.27) later.) Combining (3.25) with (3.26) and (3.27), also using inequality (2.3) with powers $\nu_{1} \leq \nu_{3} \leq \nu_{2}$ in dealing with $\|u\|_{L^{\alpha}}$, we have

$$
\frac{\lambda}{\alpha} \frac{d}{d t} \int_{U} u^{\alpha} d x+\left[(\alpha-\lambda) \frac{d_{3}}{4}-2 \varepsilon\right] \int_{U}|\nabla u|^{2-a} u^{\alpha-\lambda-1} d x \leq C_{\varepsilon}^{\prime}\|u\|_{L^{\alpha}}^{\nu_{1}}+C_{\varepsilon}^{\prime}\|u\|_{L^{\alpha}}^{\nu_{2}}+\Upsilon(t) .
$$

Choosing $\varepsilon$ sufficiently small in (3.25), we obtain (3.11).

It remains to check conditions for inequalities (3.21) and (3.27) to hold. Inequality (3.19) is valid under conditions $\mu_{2}, \mu_{3}>\alpha$ and $\alpha>2-\delta$, which, thanks to $\alpha \geq 2$, is equivalent to

$$
\alpha>\max \left\{q_{1} \lambda, q_{2}\left(\lambda-\ell_{B}\right)\right\} .
$$

The conditions for (3.27) are $\nu_{3}>\alpha, \alpha \geq 2-a$, and

$$
\alpha>\frac{n(r+a-\delta)}{2-a}=\frac{n\left(\nu_{3}-\alpha+a-\delta\right)}{2-a} .
$$

Based on definition (3.24) of $\nu_{3}$, we consider the following cases.

(i) If $\nu_{3}=\mu_{1}$, condition (3.30) becomes

$$
\alpha>n\left(\ell_{Z}-1\right) .
$$


(ii) If $\nu_{3}=\mu_{2}$, condition (3.30) becomes

$$
\alpha>\frac{-p_{1} \lambda+a-\delta}{\kappa_{f}-p_{1}} .
$$

(iii) If $\nu_{3}=\mu_{3}$, condition (3.30) becomes

$$
\alpha>\frac{p_{2}\left(-\lambda+\ell_{B}\right)+a-\delta}{\kappa_{f}-p_{2}} .
$$

(iv) If $\nu_{3}=\mu_{4}$, condition (3.30) becomes

$$
\alpha>\frac{-p_{1} \lambda+(a-\delta)}{\kappa_{B}-p_{1}} .
$$

(v) If $\nu_{3}=\mu_{5}$, condition (3.30) becomes

$$
\begin{aligned}
\alpha & >\frac{n}{2-a}\left(\frac{(2-a)\left(\left(p_{2}-1\right) \alpha+p_{2}\left(-\lambda+\ell_{B}\right)\right)+a-\delta}{1-a}+a-\delta\right) \\
& =\frac{n}{1-a}\left(\left(p_{2}-1\right) \alpha+p_{2}\left(-\lambda+\ell_{B}\right)+a-\delta\right),
\end{aligned}
$$

thus,

$$
\alpha>\frac{p_{2}\left(-\lambda+\ell_{B}\right)+a-\delta}{\kappa_{B}-p_{2}} .
$$

(vi) If $\nu_{3}=\mu_{6}$, condition (3.30) becomes

$$
\alpha>\frac{-p_{3} \lambda+a-\delta}{\kappa_{f}-p_{3}} .
$$

(vii) If $\nu_{3}=\mu_{7}$, condition (3.30) becomes

$$
\alpha>\frac{p_{4}\left(-\lambda+\ell_{f}\right)+a-\delta}{\kappa_{f}-p_{4}} .
$$

In summary, the conditions (3.29) and (3.31) -(3.37) are equivalent to $\alpha>\eta_{0}$, which is our choice of $\alpha$. The proof is complete.

We then obtain the estimates in terms of initial and boundary data in the next theorem.

Theorem 3.2. Let $\alpha, C_{0}, \theta$ be as in Lemma 3.1 and $\Upsilon(t)$ be defined by (3.8). If $T>0$ satisfies

$$
\int_{0}^{T}(1+\Upsilon(t)) d t<C_{1} \cdot\left(1+\int_{U} u_{0}(x)^{\alpha} d x\right)^{-\nu_{4}}
$$

where

$$
\nu_{4}=\nu_{4}(\alpha) \stackrel{\text { def }}{=} \frac{(2-a) \theta}{n(1-\theta)} \quad \text { and } \quad C_{1}=C_{1}(\alpha) \stackrel{\text { def }}{=} \frac{1}{4 C_{0} \nu_{4}}
$$

then for all $t \in[0, T]$

$$
\int_{U} u^{\alpha}(x, t) d x \leq\left\{\left(1+\int_{U} u_{0}(x)^{\alpha} d x\right)^{-\nu_{4}}-\frac{1}{C_{1}} \int_{0}^{t}(1+\Upsilon(\tau)) d \tau\right\}^{-\frac{1}{\nu_{4}}} .
$$


In particular, if $T>0$ satisfies

$$
\int_{0}^{T}(1+\Upsilon(t)) d t \leq C_{1} \cdot\left(1-2^{-\nu_{4}}\right)\left(1+\int_{U} u_{0}(x)^{\alpha} d x\right)^{-\nu_{4}}
$$

then

$$
\int_{U} u^{\alpha}(x, t) d x \leq 2\left(1+\int_{U} u_{0}^{\alpha}(x) d x\right) \quad \text { for all } \quad t \in[0, T]
$$

and

$$
\int_{0}^{T} \int_{U}|\nabla u|^{2-a} u^{\alpha-\lambda-1} d x d t \leq 2\left(1+1 / \nu_{4}\right)\left(1+\int_{U} u_{0}(x)^{\alpha} d x\right) .
$$

Proof. In (3.11), by applying Young's inequality to the right-hand side we find that

$$
\begin{aligned}
\frac{d}{d t} \int_{U} u^{\alpha} d x+\int_{U}|\nabla u|^{2-a} u^{\alpha-\lambda-1} d x & \leq 2 C_{0} \cdot\left(1+\|u(t)\|_{L^{\alpha}}^{\nu_{2}}+\Upsilon(t)\right) \\
& \leq 4 C_{0} \cdot(1+\Upsilon(t))\left(1+\int_{U} u^{\alpha} d x\right)^{\frac{\nu_{2}}{\alpha}} .
\end{aligned}
$$

Let $V(t)=1+\int_{U} u^{\alpha}(x, t) d x$. It follows from (3.43) that

$$
V^{\prime}(t) \leq 4 C_{0} \cdot(1+\Upsilon(t)) V(t)^{\frac{\nu_{2}}{\alpha}}=4 C_{0} \cdot(1+\Upsilon(t)) V(t)^{\nu_{4}+1} .
$$

Solving this differential inequality, under condition (3.38) yields the estimate (3.39) for all $t \in[0, T]$.

Now, let $T>0$ satisfy (3.40). Then we have from (3.39) that

$$
V(t) \leq 2 V(0) \quad \forall t \in[0, T],
$$

and hence, estimate (3.41) follows.

Next, integrating (3.43) in time from 0 to $t$ and using (3.44), (3.40) we have

$$
\begin{aligned}
& \int_{0}^{T} \int_{U}|\nabla u|^{2-a} u^{\alpha-\lambda-1} d t \leq \int_{U} u_{0}(x)^{\alpha} d x+4 C_{0} \int_{0}^{T}\left(1+\int_{U} u^{\alpha} d x\right)^{\nu_{4}+1}(1+\Upsilon(t)) d t \\
& \leq \int_{U} u_{0}(x)^{\alpha} d x+4 C_{0} \cdot\left(1+\int_{U} u_{0}(x)^{\alpha} d x\right)^{\nu_{4}+1} \int_{0}^{T}(1+\Upsilon(t)) d t \\
& \leq \int_{U} u_{0}(x)^{\alpha} d x+4 C_{0} C_{1} \cdot\left(1-2^{-\nu_{4}}\right)\left(1+\int_{U} u_{0}(x)^{\alpha} d x\right)
\end{aligned}
$$

Note that

$$
4 C_{0} C_{1} \cdot\left(1-2^{-\nu_{4}}\right)=\frac{1-2^{-\nu_{4}}}{\nu_{4}} \leq \frac{1}{\nu_{4}} .
$$

Thus we obtain (3.42) from (3.45).

\section{Maximum estimates}

In this section, we use Moser's iteration to estimate the $L^{\infty}$-norm of the solution $u(x, t)$.

For $i=1,2,3,4$, let $q_{i}$ and $p_{i}$ be as in section 3 . Assume (3.6). Let

$$
\kappa_{*}=\max \left\{\frac{(2-a) p_{1}-1}{1-a}, \frac{(2-a) p_{2}-1}{1-a}, p_{3}, p_{4}\right\},
$$




$$
\begin{aligned}
& \eta_{1}=\max \left\{\frac{p_{1} \lambda-a+\delta}{(2-a) p_{1}-1}, \frac{p_{2}\left(\lambda-\ell_{B}\right)-a+\delta}{(2-a) p_{2}-1}\right\}, \\
& \eta_{2}=\max \left\{\ell_{Z}(2-a), \ell_{f}, \frac{a-\delta+(2-a) p_{2} \ell_{B}}{1-a}\right\} .
\end{aligned}
$$

Lemma 4.1. Given $\tilde{\kappa}>\kappa_{*}$, suppose

$$
\alpha>\max \left\{2, \alpha_{*}, \eta_{1}\right\} \quad \text { and } \quad \alpha \geq \frac{\eta_{2}}{\tilde{\kappa}-\kappa_{*}} .
$$

If $T>T_{2}>T_{1} \geq 0$ then

$$
\begin{aligned}
& \sup _{t \in\left[T_{2}, T\right]} \int_{U} u^{\alpha}(x, t) d x+\int_{T_{2}}^{T} \int_{U}|\nabla u(x, t)|^{2-a} u(x, t)^{\alpha-\lambda-1} d x d t \\
& \leq c_{6}(1+T)\left(1+\frac{1}{T_{2}-T_{1}}\right) \alpha^{2} \mathcal{M}_{0}\left(\|u\|_{L^{\tilde{\kappa} \alpha}\left(U \times\left(T_{1}, T\right)\right)}^{\nu_{5}}+\|u\|_{L^{\tilde{\kappa} \alpha}\left(U \times\left(T_{1}, T\right)\right)}^{\nu_{6}}\right),
\end{aligned}
$$

where $c_{6} \geq 1$ is independent of $\alpha, \tilde{\kappa}, T, T_{1}$ and $T_{2}$,

$$
\mathcal{M}_{0}=1+\left\|\varphi_{1}\right\|_{L^{q_{1}}\left(\Gamma_{T}\right)}^{\frac{2-a}{1-a}}+\left\|\varphi_{2}\right\|_{L^{q_{2}}\left(\Gamma_{T}\right)}^{\frac{2-a}{1-a}}+\left\|f_{1}\right\|_{L^{q_{3}}\left(Q_{T}\right)}+\left\|f_{2}\right\|_{L^{q_{4}}\left(Q_{T}\right)},
$$

the positive powers $\nu_{5}$ and $\nu_{6}$ are defined by

$$
\nu_{5}=\alpha-h_{1} \quad \text { and } \quad \nu_{6}=\alpha+h_{2}
$$

with

$$
\begin{aligned}
& h_{1}=\max \left\{\lambda+1, \frac{p_{1} \lambda-a+\delta}{p_{1}(2-a)-1}, \frac{p_{2}\left(\lambda-\ell_{B}\right)-a+\delta}{p_{2}(2-a)-1}\right\}>0, \\
& h_{2}=\max \left\{0, \ell_{Z}(2-a)-\lambda-1, \ell_{f}-\lambda, \ell_{B}-\lambda, \frac{a-\delta-p_{1} \lambda}{p_{1}(2-a)-1}, \frac{a-\delta-p_{2}\left(\lambda-\ell_{B}\right)}{p_{2}(2-a)-1}\right\} \geq 0 .
\end{aligned}
$$

Proof. Denote $Q_{T}=U \times[0, T]$ and $\Gamma_{T}=\Gamma \times[0, T]$. Let $\xi=\xi(t)$ be a $C^{1}$-function on $[0, T]$ with $\xi(0)=0$ and $0 \leq \xi(t) \leq 1$. In calculations below, the generic positive constant $\bar{C}$ is independent of $\alpha, \tilde{\kappa}, T, T_{1}$ and $T_{2}$.

Multiply the PDE in (1.25) by test $u^{\alpha-\lambda} \xi^{2}$ and integrating the resulting equation over $U$ give

$$
\begin{aligned}
& \frac{\lambda}{\alpha} \frac{d}{d t} \int_{U} u^{\alpha} \xi^{2} d x-\frac{\lambda}{\alpha} \int_{U} 2 u^{\alpha} \xi \xi^{\prime} d x \\
& =\int_{U} \nabla \cdot(K(|\nabla u+Z(u)|)(\nabla u+Z(u))) u^{\alpha-\lambda} \xi^{2} d x+\int_{U} f u^{\alpha-\lambda} \xi^{2} d x .
\end{aligned}
$$

Since $\xi(t)$ is independent of $x$, same estimates as in section 3 give the following version of (3.16)

$$
\begin{aligned}
& \frac{\lambda}{\alpha} \frac{d}{d t} \int_{U} u^{\alpha} \xi^{2} d x+\frac{(\alpha-\lambda) d_{3}}{4} \int_{U}|\nabla u|^{2-a} u^{\alpha-\lambda-1} \xi^{2} d x \leq C \alpha \int_{U}\left(u^{\nu_{1}}+u^{\mu_{1}}\right) \xi^{2} d x \\
& +\int_{\Gamma} u^{\alpha-\lambda}\left(\varphi_{1}+\varphi_{2} u^{\ell_{B}}\right) \xi^{2} d \sigma+\int_{U}\left(f_{1}+f_{2} u^{\ell_{f}}\right) u^{\alpha-\lambda} \xi^{2} d x+\frac{\lambda}{\alpha} \int_{U} 2 u^{\alpha} \xi \xi^{\prime} d x .
\end{aligned}
$$

Integrating the previous inequality in time and applying Hölder's inequality give

$$
\begin{aligned}
\frac{\lambda}{\alpha} \sup _{t \in[0, T]} \int_{U} u^{\alpha}(x, t) \xi^{2}(t) d x+ & \frac{(\alpha-\lambda) d_{3}}{4} \iint_{Q_{T}}|\nabla u|^{2-a} u^{\alpha-\lambda-1} \xi^{2} d x d t \\
& \leq C \alpha \iint_{Q_{T}}\left(u^{\nu_{1}}+u^{\mu_{1}}\right) \xi^{2} d x d t+\frac{\lambda}{\alpha} \iint_{Q_{T}} 2 u^{\alpha} \xi \xi^{\prime} d x d t+\sum_{i=1}^{4} I_{i}
\end{aligned}
$$


where

$$
\begin{aligned}
I_{i} & =\left(\iint_{\Gamma_{T}} u^{\alpha_{i}} \xi^{2} d \sigma d t\right)^{1 / p_{i}} F_{i} \quad \text { with } F_{i}=\left(\iint_{\Gamma_{T}} \varphi_{i}^{q_{i}} \xi^{2} d \sigma d t\right)^{1 / q_{i}} \text { for } i=1,2, \\
I_{3} & =\left(\iint_{Q_{T}} u^{\alpha_{3}} \xi^{2} d x d t\right)^{1 / p_{3}} F_{3} \quad \text { with } F_{3}=\left(\iint_{Q_{T}} f_{1}^{q_{3}} \xi^{2} d x d t\right)^{1 / q_{3}}, \\
I_{4} & =\left(\iint_{Q_{T}} u^{\alpha_{4}} \xi^{2} d x d t\right)^{1 / p_{4}} F_{4} \quad \text { with } F_{4}=\left(\iint_{Q_{T}} f_{2}^{q_{4}} \xi^{2} d x d t\right)^{1 / q_{4}},
\end{aligned}
$$

with

$$
\begin{aligned}
& \alpha_{1}=p_{1}(\alpha-\lambda)>p_{1}>1, \\
& \alpha_{2}=p_{2}\left(\alpha-\lambda+\ell_{B}\right)>p_{2}\left(1+\ell_{B}\right)>1, \\
& \alpha_{3}=p_{3}(\alpha-\lambda)>p_{3}>1 \text {, } \\
& \alpha_{4}=p_{4}\left(\alpha-\lambda+\ell_{f}\right)>p_{4}\left(1+\ell_{f}\right)>1 \text {. }
\end{aligned}
$$

For $I_{1}$, by using the trace theorem (2.5), we have

$$
\iint_{\Gamma_{T}} u^{\alpha_{1}} \xi^{2} d \sigma d t \leq c_{1} \iint_{Q_{T}} u^{\alpha_{1}} \xi^{2} d x d t+c_{2} \alpha_{1} \iint_{Q_{T}} u^{\alpha_{1}-1}|\nabla u| \xi^{2} d x d t
$$

In the last integral, writing $u^{\alpha_{1}-1}|\nabla u|$ as a product of $u^{\frac{\alpha-\lambda-1}{2-a}}|\nabla u|$ and $u^{\alpha_{1}-\frac{\alpha-a+\delta}{2-a}}$, and applying Hölder's inequality with powers $2-a$ and $(2-a) /(1-a)$, we obtain

$$
\begin{aligned}
\iint_{\Gamma_{T}} u^{\alpha_{1}} \xi^{2} d \sigma d t \leq c_{1} \iint_{Q_{T}} & u^{\alpha_{1}} \xi^{2} d x d t \\
& +c_{2} \alpha_{1}\left(\iint_{Q_{T}} u^{\alpha-\lambda-1}|\nabla u|^{2-a} \xi^{2} d x d t\right)^{\frac{1}{2-a}}\left(\iint_{Q_{T}} u^{m_{1}} \xi^{2} d x d t\right)^{\frac{1-a}{2-a}},
\end{aligned}
$$

where

$$
m_{1}=\frac{(2-a) \alpha_{1}-\alpha+a-\delta}{1-a}=\frac{\left((2-a) p_{1}-1\right) \alpha-p_{1} \lambda+a-\delta}{1-a}>0 .
$$

Denote $X(\alpha)=\iint_{Q_{T}} u^{\alpha}(x, t) \xi(t) d x d t$. We will use the fact $0 \leq \xi^{2} \leq \xi \leq 1$. By (4.8) and Young's inequality

$$
\begin{aligned}
I_{1} \leq & c_{1}^{1 / p_{1}} X\left(\alpha_{1}\right)^{1 / p_{1}} F_{1}+\left(c_{2} \alpha_{1}\right)^{1 / p_{1}}\left(\iint_{Q_{T}} u^{\alpha-\lambda-1}|\nabla u|^{2-a} d x d t\right)^{\frac{1}{p_{1}(2-a)}} X\left(m_{1}\right)^{\frac{1-a}{p_{1}(2-a)}} F_{1} \\
\leq & c_{1}^{1 / p_{1}} X\left(\alpha_{1}\right)^{1 / p_{1}} F_{1}+\varepsilon \iint_{Q_{T}} u^{\alpha-\lambda-1}|\nabla u|^{2-a} d x d t \\
& +\left[\varepsilon^{-1}\left(c_{2} \alpha_{1}\right)^{2-a}\right]^{\frac{1}{p_{1}(2-a)-1}} X\left(m_{1}\right)^{\frac{1-a}{p_{1}(2-a)-1}} F_{1}^{\frac{p_{1}(2-a)}{p^{(2-a)-1}}} .
\end{aligned}
$$

Similarly,

$$
\begin{aligned}
I_{2} \leq & c_{3} X\left(\alpha_{2}\right)^{1 / p_{2}} F_{2}+\varepsilon \iint_{Q_{T}} u^{\alpha-\lambda-1}|\nabla u|^{2-a} d x d t \\
& +\left[\varepsilon^{-1}\left(c_{4} \alpha_{2}\right)^{2-a}\right]^{\frac{1}{p_{2}(2-a)-1}} X\left(m_{2}\right)^{\frac{1-a}{p_{2}(2-a)-1}} F_{2}^{\frac{p_{2}(2-a)}{p_{2}(2-a)-1}},
\end{aligned}
$$

where

$$
m_{2}=\frac{(2-a) \alpha_{2}-\alpha+a-\delta}{1-a}=\frac{\left((2-a) p_{2}-1\right) \alpha+p_{2}\left(\ell_{B}-\lambda\right)+a-\delta}{1-a}>0 .
$$


We now choose $\xi(t)$ such that

$$
\xi(t)=0 \text { for } 0 \leq t \leq T_{1}, \xi(t)=1 \text { for } T_{2} \leq t \leq T \text {, and } 0 \leq \xi^{\prime}(t) \leq \frac{2}{T_{2}-T_{1}} \text { for } 0 \leq t \leq T \text {. }
$$

Then

$$
\frac{\lambda}{\alpha} \iint_{Q_{T}} 2 u^{\alpha} \xi \xi^{\prime} d x d t \leq \frac{\bar{C}}{T_{2}-T_{1}} X(\alpha) .
$$

Therefore, with $\varepsilon=(\alpha-\lambda) d_{3} / 16$, it follows (4.7) and the above estimates (4.9), (4.10), (4.12) that

$$
\begin{aligned}
& \frac{\lambda}{\alpha} \sup _{t \in[0, T]} \int_{U} u^{\alpha}(x, t) \xi^{2}(t) d x+\frac{(\alpha-\lambda) d_{3}}{8} \iint_{Q_{T}}|\nabla u|^{2-a} u^{\alpha-\lambda-1} \xi^{2} d x d t \leq \bar{C} \alpha\left(X\left(\mu_{1}\right)+X\left(\nu_{1}\right)\right) \\
& +\frac{\bar{C}}{T_{2}-T_{1}} X(\alpha)+\bar{C} \sum_{i=1}^{4} X\left(\alpha_{i}\right)^{1 / p_{i}} F_{i}+\bar{C} \sum_{j=1}^{2}\left(\frac{\alpha_{j}^{2-a}}{\alpha-\lambda}\right)^{\frac{1}{p_{j}(2-a)-1}} X\left(m_{j}\right)^{\frac{1-a}{p_{j}(2-a)-1}} F_{j}^{\frac{p_{j}(2-a)}{p_{j}(2-a)-1}}
\end{aligned}
$$

We bound the left-hand side of (4.13) from below by

$$
I_{0} \stackrel{\text { def }}{=} \frac{\lambda}{\alpha} \sup _{t \in[0, T]} \int_{U} u^{\alpha}(x, t) \xi^{2}(t) d x+\frac{d_{3}}{8} \iint_{Q_{T}}|\nabla u|^{2-a} u^{\alpha-\lambda-1} \xi^{2} d x d t .
$$

We now bound the right-hand side of (4.13). We will estimate many $X(\cdot)$-terms by using

$$
J_{0} \stackrel{\text { def }}{=}\|u\|_{L^{\tilde{\kappa} \alpha}\left(U \times\left(T_{1}, T\right)\right)} .
$$

Claim. Under condition (4.2), one has

$$
0<\mu_{1}, \alpha_{1}, \alpha_{2}, \alpha_{3}, \alpha_{4}, m_{1}, m_{2} \leq \tilde{\kappa} \alpha .
$$

We accept this claim at the moment. By Hölder's inequality if $0<\beta \leq \tilde{\kappa} \alpha$ then

$$
X(\beta) \leq \int_{T_{1}}^{T} \int_{U} u^{\beta} d x d t \leq J_{0}^{\beta}\left|Q_{T}\right|^{1-\frac{\beta}{\kappa \alpha}} \leq J_{0}^{\beta}\left(1+\left|Q_{T}\right|\right) .
$$

Note for $j=1,2$ that

$$
\frac{1-a}{p_{j}(2-a)-1}<1 \text { and } \frac{p_{j}(2-a)}{p_{j}(2-a)-1}<\frac{2-a}{1-a} .
$$

Using also $\alpha-\lambda \geq \alpha / 2$ and $\alpha_{1}, \alpha_{2} \leq\left(p_{1}+p_{2}\right)\left(1+\ell_{B}\right) \alpha$, then for $j=1,2$,

$$
\left(\frac{\alpha_{j}^{2-a}}{\alpha-\lambda}\right)^{\frac{1}{p_{j}(2-a)-1}} \leq\left(2\left[\left(p_{1}+p_{2}\right)\left(1+\ell_{B}\right)\right]^{2-a} \alpha^{1-a}\right)^{\frac{1}{p_{j}(2-a)-1}} \leq C \alpha .
$$

Thus, we have from (4.13), 4.15) that

$$
I_{0} \leq \bar{C}\left(1+\left|Q_{T}\right|\right)\left(\alpha J_{0}^{\mu_{1}}+\alpha J_{0}^{\nu_{1}}+\frac{1}{T_{2}-T_{1}} J_{0}^{\alpha}+\sum_{i=1}^{4} J_{0}^{\frac{\alpha_{i}}{p_{i}}} F_{i}+\alpha \sum_{j=1}^{2} J_{0}^{\frac{m_{j}(1-a)}{p_{j}(2-a)-1}} F_{j}^{\frac{p_{j}(2-a)}{p_{j}(2-a)-1}}\right) .
$$

Calculating the powers of $J_{0}$ gives

$$
\frac{\alpha_{1}}{p_{1}}=\frac{\alpha_{3}}{p_{3}}=\alpha-\lambda, \quad \frac{\alpha_{2}}{p_{2}}=\alpha-\lambda+\ell_{B}, \quad \frac{\alpha_{4}}{p_{4}}=\alpha-\lambda+\ell_{f},
$$




$$
\frac{m_{1}(1-a)}{p_{1}(2-a)-1}=\alpha+\frac{-p_{1} \lambda+a-\delta}{p_{1}(2-a)-1}, \quad \frac{m_{2}(1-a)}{p_{2}(2-a)-1}=\alpha+\frac{p_{2}\left(\ell_{B}-\lambda\right)+a-\delta}{p_{2}(2-a)-1} .
$$

Hence,

$$
I_{0} \leq \bar{C}\left(1+\left|Q_{T}\right|\right) \alpha\left(1+\frac{1}{T_{2}-T_{1}}\right) \tilde{F} \cdot \tilde{J}
$$

where

$$
\begin{aligned}
\tilde{F}= & 1+F_{1}+F_{2}+F_{1}^{\frac{p_{1}(2-a)}{p_{1}(2-a)-1}}+F_{2}^{\frac{p_{2}(2-a)}{p_{2}(2-a)-1}}+F_{3}+F_{4}, \\
\tilde{J}= & J_{0}^{\alpha+\ell_{Z}(2-a)-\lambda-1}+J_{0}^{\alpha-\lambda-1}+J_{0}^{\alpha}+J_{0}^{\alpha-\lambda}+J_{0}^{\alpha+\frac{-p_{1} \lambda+a-\delta}{p_{1}(2-a)-1}}+J_{0}^{\alpha+\ell_{B}-\lambda} \\
& +J_{0}^{\alpha+\frac{p_{2}\left(\ell_{B}-\lambda\right)+a-\delta}{p_{2}(2-a)-1}}+J_{0}^{\alpha+\ell_{f}-\lambda} .
\end{aligned}
$$

Using the second inequality in (4.16) and inequality (2.4), we simply estimate

$$
\tilde{F} \leq 3\left(1+F_{1}^{\frac{2-a}{1-a}}+F_{2}^{\frac{2-a}{1-a}}+F_{3}+F_{4}\right) \leq 3 \mathcal{M}_{0} .
$$

Also, by using (2.3),

$$
\tilde{J} \leq 8\left(J_{0}^{\alpha-h_{1}}+J_{0}^{\alpha+h_{2}}\right) .
$$

Therefore

$$
I_{0} \leq \bar{C}\left(1+\left|Q_{T}\right|\right) \alpha\left(1+\frac{1}{T_{2}-T_{1}}\right) \mathcal{M}_{0}\left(J_{0}^{\alpha-h_{1}}+J_{0}^{\alpha+h_{2}}\right) .
$$

By (4.17) and definition of $I_{0}$ we have

$$
\begin{aligned}
& \sup _{t \in[0, T]} \int_{U} u^{\alpha}(x, t) \xi^{2}(t) d x+\iint_{Q_{T}}|\nabla u|^{2-a} u^{\alpha+\lambda-1} \xi^{2} d x d t \\
& \leq \bar{C}\left(1+\left|Q_{T}\right|\right) \alpha^{2}\left(1+\frac{1}{T_{2}-T_{1}}\right) \mathcal{M}_{0}\left(J_{0}^{1-h_{1} / \alpha}+J_{0}^{1+h_{2} / \alpha}\right) .
\end{aligned}
$$

This estimate, together with property (4.11) of function $\xi(t)$, and the fact $1+\left|Q_{T}\right| \leq(1+$ $|U|)(1+T)$, implies (4.3).

Finally, we verify the claim (4.14). Dividing (4.14) by $\alpha$ gives an equivalent statement

$$
\begin{aligned}
1+ & \frac{\ell_{Z}(2-a)-\lambda-1}{\alpha}, p_{1}-\frac{\lambda}{\alpha}, p_{2}+\frac{\ell_{B}-\lambda}{\alpha}, p_{3}-\frac{\lambda}{\alpha}, p_{4}+\frac{\ell_{f}-\lambda}{\alpha}, \\
& \quad \frac{(2-a) p_{1}-1}{1-a}+\frac{-(2-a) p_{1} \lambda+a-\delta}{\alpha(1-a)}, \frac{(2-a) p_{2}-1}{1-a}+\frac{(2-a) p_{2}\left(\ell_{B}-\lambda\right)+a-\delta}{\alpha(1-a)} \leq \tilde{\kappa} .
\end{aligned}
$$

Note for $i=1,2$ that $p_{i} \leq \frac{(2-a) p_{i}-1}{1-a}$. Then by definition (4.1) of $\kappa_{*}$,

$$
1, p_{1}, p_{2}, p_{3}, p_{4}, \frac{(2-a) p_{1}-1}{1-a}, \frac{(2-a) p_{2}-1}{1-a} \leq \kappa_{*} .
$$

Hence, we can bound the left-hand side of (4.18) by

$$
\begin{aligned}
& \kappa_{*}+\max \left\{\frac{\ell_{Z}(2-a)-\lambda-1}{\alpha}, \frac{\ell_{B}-\lambda}{\alpha}, \frac{\ell_{f}-\lambda}{\alpha}, \frac{a-\delta-(2-a) p_{1} \lambda}{\alpha(1-a)}, \frac{a-\delta+(2-a) p_{2}\left(\ell_{B}-\lambda\right)}{\alpha(1-a)}\right\} \\
& \leq \kappa_{*}+\frac{\eta_{2}}{\alpha} .
\end{aligned}
$$

Since $\tilde{\kappa}>\kappa_{*}$, we choose a sufficient condition for (4.18) to be $\eta_{2} / \alpha \leq \tilde{\kappa}-\kappa_{*}$, which is satisfied by (4.2). The proof is complete. 
Lemma 4.2. Let $\tilde{\kappa}, \alpha, \nu_{5}, \nu_{6}$ and $\mathcal{M}_{0}$ be as in Lemma 4.1. If $T>T_{2}>T_{1} \geq 0$ then

$$
\|u\|_{L^{\kappa \alpha}\left(U \times\left(T_{2}, T\right)\right)} \leq A_{\alpha}^{\frac{1}{\alpha}}\left(\|u\|_{L^{\tilde{\kappa} \alpha}\left(U \times\left(T_{1}, T\right)\right)}^{\nu^{r}}+\|u\|_{L^{\tilde{\kappa} \alpha}\left(U \times\left(T_{1}, T\right)\right)}^{\nu_{7}}\right)^{\frac{1}{\alpha}},
$$

where $\kappa$ is defined by (2.16),

$$
\nu_{7}=\nu_{7}(\alpha) \stackrel{\text { def }}{=} \frac{\alpha \nu_{6}}{\alpha+\delta-a}
$$

and

$$
A_{\alpha}=c_{7}(1+T)^{2}\left(1+\frac{1}{T_{2}-T_{1}}\right)^{2} \alpha^{6-a} \mathcal{M}_{0}^{2}
$$

with $c_{7} \geq 1$ independent of $\alpha, \tilde{\kappa}, T, T_{1}$ and $T_{2}$.

Proof. We follow the proof of Proposition 6.2 in [4]. We use Sobolev inequality (2.15) in Lemma 2.3

$$
\|u\|_{L^{\kappa \alpha}\left(U \times\left(T_{2}, T\right)\right)} \leq \hat{C}^{\frac{1}{\kappa \alpha}}\left\{I^{\tilde{\theta}} \cdot \sup _{t \in\left[T_{2}, T\right]}\left(\int_{U}|u(x, t)|^{\alpha} d x\right)^{1-\tilde{\theta}}\right\}^{\frac{1}{\alpha}}
$$

where $\hat{C}=c_{5} \alpha^{2-a}$ with $c_{5} \geq 1$, the numbers $\tilde{\theta}$ and $\kappa$ are defined in (2.16) and

$$
I=\left[\int_{T_{2}}^{T} \int_{U}|u|^{\alpha+\delta-a} d x d t+\int_{T_{2}}^{T} \int_{U}|u|^{\alpha-\lambda-1}|\nabla u|^{2-a} d x d t\right]^{\frac{\alpha}{\alpha+\delta-a}}
$$

Note that

$$
\frac{\alpha}{\alpha+\delta-a} \leq 2 \text { and } 2^{\frac{\alpha}{\alpha+\delta-a}-1} \leq 2
$$

Then applying inequality (2.2), we find that

$$
I \leq 2\left(\int_{T_{2}}^{T} \int_{U}|u|^{\alpha+\delta-a} d x d t\right)^{\frac{\alpha}{\alpha+\delta-a}}+2\left(\int_{T_{2}}^{T} \int_{U}|u|^{\alpha-\lambda-1}|\nabla u|^{2-a} d x d t\right)^{\frac{\alpha}{\alpha+\delta-a}} .
$$

Applying Hölder's inequality to the first integral on the right-hand side of (4.26) with conjugate exponents $\frac{\tilde{\kappa} \alpha}{\alpha+\delta-a}$ and $\frac{\tilde{\kappa} \alpha}{(\tilde{\kappa}-1) \alpha+a-\delta}$, we get

$$
I \leq 2\left|Q_{T}\right|^{\left(1-\frac{\alpha+\delta-a}{\tilde{\kappa} \alpha}\right) \frac{\alpha}{\alpha+\delta-a}}\left(\int_{T_{2}}^{T} \int_{U}|u|^{\tilde{\kappa} \alpha} d x d t\right)^{\frac{1}{\tilde{\kappa}}}+2\left(\int_{T_{2}}^{T} \int_{U}|u|^{\alpha-\lambda-1}|\nabla u|^{2-a} d x d t\right)^{\frac{\alpha}{\alpha+\delta-a}} .
$$

Next, we use (4.3) to estimate the second term on the right-hand side of (4.27). We denote

$$
\mathcal{S}=\|u\|_{L^{\tilde{\kappa} \alpha}\left(U \times\left(T_{1}, T\right)\right)} \quad \text { and } \quad \mathcal{M}=c_{6}(1+T)\left(1+\frac{1}{T_{2}-T_{1}}\right) \alpha^{2} \mathcal{M}_{0}
$$

where $c_{6}$ is the positive constant in (4.3). Then combining (4.24) and (4.27) yields

$$
\begin{aligned}
\|u\|_{L^{\kappa \alpha}\left(U \times\left(T_{2}, T\right)\right)} & \leq \hat{C}^{\frac{1}{\kappa \alpha}}\left\{\left[2\left(1+\left|Q_{T}\right|\right)^{\frac{\alpha}{\alpha+\delta-a}} \mathcal{S}^{\alpha}+2\left(\mathcal{M}\left(\mathcal{S}^{\nu_{5}}+\mathcal{S}^{\nu_{6}}\right)\right)^{\frac{\alpha}{\alpha+\delta-a}}\right]^{\tilde{\theta}}\left(\mathcal{M}\left(\mathcal{S}^{\nu_{5}}+\mathcal{S}^{\nu_{6}}\right)\right)^{1-\tilde{\theta}}\right\}^{\frac{1}{\alpha}} \\
& \leq \hat{C}^{\frac{1}{\kappa \alpha}}\left\{\left[2\left(1+\left|Q_{T}\right|\right)^{2} \mathcal{S}^{\alpha}+2\left(\mathcal{M}\left(\mathcal{S}^{\nu_{5}}+\mathcal{S}^{\nu_{6}}\right)\right)^{\frac{\alpha}{\alpha+\delta-a}}+\mathcal{M}\left(\mathcal{S}^{\nu_{5}}+\mathcal{S}^{\nu_{6}}\right)\right]^{\tilde{\theta}+1-\tilde{\theta}}\right\}^{\frac{1}{\alpha}} \\
& =\hat{C}^{\frac{1}{\kappa \alpha}}\left\{2\left(1+\left|Q_{T}\right|\right)^{2} \mathcal{S}^{\alpha}+2 \mathcal{M}^{\frac{\alpha}{\alpha+\delta-a}}\left(\mathcal{S}^{\nu_{5}}+\mathcal{S}^{\nu_{6}}\right)^{\frac{\alpha}{\alpha+\delta-a}}+\mathcal{M}\left(\mathcal{S}^{\nu_{5}}+\mathcal{S}^{\nu_{6}}\right)\right\}^{\frac{1}{\alpha}} .
\end{aligned}
$$

Since $\nu_{5}<\alpha<\nu_{6}$, we use (2.3) to estimate $\mathcal{S}^{\alpha} \leq \mathcal{S}^{\nu_{5}}+\mathcal{S}^{\nu_{6}}$. Also, by (2.2) and (4.25),

$$
\left(\mathcal{S}^{\nu_{5}}+\mathcal{S}^{\nu_{6}}\right)^{\frac{\alpha}{\alpha+\delta-a}} \leq 2\left(\mathcal{S}^{\frac{\nu_{5} \alpha}{\alpha+\delta-a}}+\mathcal{S}^{\frac{\nu_{6} \alpha}{\alpha+\delta-a}}\right)
$$


Thus, we have

$$
\|u\|_{L^{\kappa \alpha}\left(U \times\left(T_{2}, T\right)\right)} \leq \hat{C}^{\frac{1}{\kappa \alpha}}\left\{\left(2\left(1+\left|Q_{T}\right|\right)^{2}+\mathcal{M}\right)\left(\mathcal{S}^{\nu_{5}}+\mathcal{S}^{\nu_{6}}\right)+4 \mathcal{M}^{\frac{\alpha}{\alpha+\delta-a}}\left(\mathcal{S}^{\frac{\nu_{5} \alpha}{\alpha+\delta-a}}+\mathcal{S}^{\frac{\nu_{6} \alpha}{\alpha+\delta-a}}\right)\right\}^{\frac{1}{\alpha}} .
$$

Using $\mathcal{S}^{\nu_{6}}, \mathcal{S}^{\frac{\nu_{5} \alpha}{\alpha+\delta-a}} \leq \mathcal{S}^{\nu_{5}}+\mathcal{S}^{\frac{\nu_{6} \alpha}{\alpha+\delta-a}}$, we obtain

$$
\|u\|_{L^{\kappa \alpha}\left(U \times\left(T_{2}, T\right)\right)} \leq \hat{C}^{\frac{1}{\kappa \alpha}}\left\{8\left(\left(1+\left|Q_{T}\right|\right)^{2}+\mathcal{M}+\mathcal{M}^{\frac{\alpha}{\alpha+\delta-a}}\right)\left(\mathcal{S}^{\nu_{5}}+\mathcal{S}^{\frac{\nu_{6} \alpha}{\alpha+\delta-a}}\right)\right\}^{\frac{1}{\alpha}} .
$$

Note also that $\hat{C}^{\frac{1}{\kappa}} \leq \hat{C}$, then we obtain from (4.29) that

$$
\|u\|_{L^{\kappa \alpha}\left(U \times\left(T_{2}, T\right)\right)} \leq \mathcal{M}_{1}^{\frac{1}{\alpha}}\left(\|u\|_{L^{\tilde{\kappa} \alpha}\left(U \times\left(T_{1}, T\right)\right)}^{\nu_{5}}+\|u\|_{L^{\tilde{\kappa} \alpha}\left(U \times\left(T_{1}, T\right)\right)}^{\nu_{7}}\right)^{\frac{1}{\alpha}},
$$

where

$$
\mathcal{M}_{1}=8 c_{5} \alpha^{2-a}\left[\left(1+\left|Q_{T}\right|\right)^{2}+\mathcal{M}+\mathcal{M}^{\frac{\alpha}{\alpha+\delta-a}}\right] .
$$

We estimate $\mathcal{M}_{1}$. Note that $\mathcal{M}_{0}, \mathcal{M}, \mathcal{M}_{1} \geq 1$. Recall from (4.25) that the last power of $\mathcal{M}$ in (4.31) is less than or equal to 2 . Then

$$
\begin{aligned}
\mathcal{M}_{1} & \leq 8 c_{5} \alpha^{2-a}\left((1+|U|)^{2}(1+T)^{2}+2 \mathcal{M}^{2}\right) \\
& \leq 8 c_{5} \alpha^{2-a}\left((1+|U|)^{2}(1+T)^{2}+2\left\{c_{6} \alpha^{2}\left(1+\frac{1}{T_{2}-T_{1}}\right)(1+T) \mathcal{M}_{0}\right\}^{2}\right) \\
& \leq 8 c_{5}\left((1+|U|)^{2}+2 c_{6}^{2}\right) \alpha^{6-a}\left(1+\frac{1}{T_{2}-T_{1}}\right)^{2}(1+T)^{2} \mathcal{M}_{0}^{2} .
\end{aligned}
$$

Hence we obtain (4.21) from (4.30) and (4.32).

Next, we apply Moser's iteration. For that we recall the following lemma on finding the upper bounds of certain numeric sequences.

Lemma 4.3 ( [4], Lemma A.2). Let $y_{j} \geq 0, \kappa_{j}>0, s_{j} \geq r_{j}>0$ and $\omega_{j} \geq 1$ for all $j \geq 0$. Suppose there is $A \geq 1$ such that

$$
y_{j+1} \leq A^{\frac{\omega_{j}}{\kappa_{j}}}\left(y_{j}^{r_{j}}+y_{j}^{s_{j}}\right)^{\frac{1}{\kappa_{j}}} \quad \forall j \geq 0 .
$$

Denote $\beta_{j}=r_{j} / \kappa_{j}$ and $\gamma_{j}=s_{j} / \kappa_{j}$. Assume

$$
\bar{\alpha} \stackrel{\text { def }}{=} \sum_{j=0}^{\infty} \frac{\omega_{j}}{\kappa_{j}}<\infty \text { and the products } \prod_{j=0}^{\infty} \beta_{j}, \prod_{j=0}^{\infty} \gamma_{j} \text { converge to positive numbers } \bar{\beta}, \bar{\gamma} \text {, resp. }
$$

Then

$$
y_{j} \leq(2 A)^{G_{j} \bar{\alpha}} \max \left\{y_{0}^{\gamma_{0} \ldots \gamma_{j-1}}, y_{0}^{\beta_{0} \ldots \beta_{j-1}}\right\} \quad \forall j \geq 1,
$$

where $G_{j}=\max \left\{1, \gamma_{m} \gamma_{m+1} \ldots \gamma_{n}: 1 \leq m \leq n<j\right\}$. Consequently,

$$
\limsup _{j \rightarrow \infty} y_{j} \leq(2 A)^{G \bar{\alpha}} \max \left\{y_{0}^{\bar{\gamma}}, y_{0}^{\bar{\beta}}\right\}, \quad \text { where } G=\limsup _{j \rightarrow \infty} G_{j}
$$


For iterations below, we will require

$$
\kappa_{*}^{2}<\kappa_{f}
$$

which is equivalent to

$$
p_{1}, p_{2}<\frac{\sqrt{\kappa_{f}}+1}{2-a} \text { and } p_{3}, p_{4}<\sqrt{\kappa_{f}} .
$$

Therefore, we assume (4.35) for the remainder of this section. We now fix $\tilde{\kappa}$ such that

$$
\kappa_{*}<\tilde{\kappa}<\sqrt{\kappa_{f}}
$$

For simplicity, we take

$$
\tilde{\kappa}=\sqrt{\frac{\kappa_{*}^{2}+\kappa_{f}}{2}} .
$$

Theorem 4.4. Let $\alpha_{0}$ be a positive number such that

$$
\alpha_{0}>\max \left\{2, \alpha_{*}, \eta_{1}\right\} \quad \text { and } \quad \alpha_{0} \geq \max \left\{\frac{\eta_{2}}{\tilde{\kappa}-\kappa_{*}}, \frac{a-\delta}{\kappa_{f}-\tilde{\kappa}^{2}}\right\} .
$$

Then there are positive constants $C, \tilde{\mu}, \tilde{\nu}, \omega_{1}, \omega_{2}, \omega_{3}$ such that if $T>0$ and $\sigma \in(0,1)$ then

$$
\|u\|_{L^{\infty}(U \times(\sigma T, T))} \leq C\left(1+\frac{1}{\sigma T}\right)^{\omega_{1}}(1+T)^{\omega_{2}} \mathcal{M}_{0}^{\omega_{3}} \max \left\{\|u\|_{L^{\tilde{\kappa} \alpha_{0}(U \times(0, T))}}^{\tilde{\tilde{n}}},\|u\|_{L^{\tilde{\kappa} \alpha_{0}(U \times(0, T))}}^{\tilde{\tilde{N}}}\right\},
$$

where $\mathcal{M}_{0}$ is defined by 4.4).

Proof. We set up to iterate inequality (4.21) in Lemma 4.2, Note that $\alpha=\alpha_{0}$ satisfies (4.2). Define for $j \geq 0, \beta_{j}=\tilde{\kappa}^{j} \alpha_{0}$. Since $\tilde{\kappa}>1$, the sequence $\left\{\beta_{j}\right\}_{j=0}^{\infty}$ is increasing, and hence, so is the sequence $\left\{\kappa\left(\beta_{j}\right)\right\}_{j=0}^{\infty}$. We then have from the last fact and the choice of $\alpha_{0}$ that

$$
\kappa\left(\beta_{j}\right) \geq \kappa\left(\alpha_{0}\right)=\kappa_{f}-\frac{a-\delta}{\alpha_{0}} \geq \tilde{\kappa}^{2} .
$$

For $j \geq 0$, let $t_{j}=\sigma T\left(1-\frac{1}{2^{j}}\right)$. For $j \geq 0$, applying (4.21) of Theorem4.2 with $\alpha=\beta_{j}, T_{2}=t_{j+1}$ and $T_{1}=t_{j}$, we have

$$
\begin{aligned}
\|u\|_{L^{\kappa\left(\beta_{j}\right) \beta_{j}\left(U \times\left(t_{j+1}, T\right)\right)}} & \leq A_{\beta_{j}}^{\frac{1}{\beta_{j}}}\left(\|u\|_{L^{\tilde{\kappa} \beta_{j}}\left(U \times\left(t_{j}, T\right)\right)}^{\nu_{5}\left(\beta_{j}\right)}+\|u\|_{L^{\tilde{\kappa} \beta_{j}}\left(U \times\left(t_{j}, T\right)\right)}^{\nu_{7}\left(\beta_{j}\right)}\right)^{\frac{1}{\beta_{j}}} \\
& =A_{\beta_{j}}^{\frac{1}{\beta_{j}}}\left(\|u\|_{L^{\beta_{j+1}\left(U \times\left(t_{j}, T\right)\right)}}^{\tilde{r}_{j}}+\|u\|_{L^{\beta_{j+1}\left(U \times\left(t_{j}, T\right)\right)}}^{\tilde{s}_{j}}\right)^{\frac{1}{\beta_{j}}}
\end{aligned}
$$

where $\tilde{r}_{j}=\nu_{5}\left(\beta_{j}\right)$ and $\tilde{s}_{j}=\nu_{7}\left(\beta_{j}\right)$, see formula (4.22). Note from (4.39) that

$$
\kappa\left(\beta_{j}\right) \beta_{j} \geq \tilde{\kappa}^{2} \beta_{j}=\beta_{j+2} .
$$

Define for $j \geq 0$ that $\mathcal{Q}_{j}=U \times\left(t_{j}, T\right)$ and $Y_{j}=\|u\|_{L^{\beta_{j+1}\left(\mathcal{Q}_{j}\right)}}$. By Hölder's inequality

$$
Y_{j+1}=\|u\|_{L^{\beta_{j+2}\left(\mathcal{Q}_{j+1}\right)}} \leq\left|\mathcal{Q}_{j+1}\right|^{\frac{1}{\beta_{j+2}}-\frac{1}{\kappa\left(\beta_{j}\right) \beta_{j}}}\|u\|_{L^{\kappa\left(\beta_{j}\right) \beta_{j}}\left(\mathcal{Q}_{j+1}\right)} \leq\left(1+\left|\mathcal{Q}_{0}\right|\right)^{\frac{1}{\beta_{j}}}\|u\|_{L^{\kappa\left(\beta_{j}\right) \beta_{j}}\left(\mathcal{Q}_{j+1}\right)} .
$$

Combining inequalities (4.40) and (4.41) gives

$$
Y_{j+1} \leq \widehat{A}_{j}^{\frac{1}{\beta_{j}}}\left(Y_{j}^{\tilde{r}_{j}}+Y_{j}^{\tilde{s}_{j}}\right)^{\frac{1}{\beta_{j}}} \quad \forall j \geq 0, \quad \text { where } \widehat{A}_{j}=\left(1+\left|\mathcal{Q}_{0}\right|\right) A_{\beta_{j}}
$$


Now we estimate $\widehat{A}_{j}$. Note that $\left|\mathcal{Q}_{0}\right|=\left|Q_{T}\right| \leq(1+|U|)(1+T)$. From (4.23) and (4.42), we have

$$
\begin{aligned}
\widehat{A}_{j} & \leq c_{7}(1+|U|) \beta_{j}^{6-a}\left(1+\frac{2^{j+1}}{\sigma T}\right)^{2}(1+T)^{3} \mathcal{M}_{0}^{2} \\
& \leq c_{7}(1+|U|)\left(\tilde{\kappa}^{j} \alpha_{0}\right)^{6-a} 4^{j+1}\left(1+\frac{1}{\sigma T}\right)^{2}(1+T)^{3} \mathcal{M}_{0}^{2} \leq A_{T, \sigma, \alpha_{0}}^{j+1}
\end{aligned}
$$

where

$$
A_{T, \sigma, \alpha_{0}}=\max \left\{4 \tilde{\kappa}^{6-a}, 4 c_{7}(1+|U|) \alpha_{0}^{6-a}\left(1+\frac{1}{\sigma T}\right)^{2}(1+T)^{3} \mathcal{M}_{0}^{2}\right\} \geq 1
$$

Hence

$$
Y_{j+1} \leq A_{T, \sigma, \alpha_{0}}^{\frac{j+1}{\beta_{j}}}\left(Y_{j}^{\tilde{r}_{j}}+Y_{j}^{\tilde{s}_{j}}\right)^{\frac{1}{\beta_{j}}}
$$

We have

$$
\sum_{j=0}^{\infty} \frac{j+1}{\beta_{j}}=\frac{1}{\alpha_{0}} \sum_{j=0}^{\infty} \frac{j+1}{\tilde{\kappa}^{j}}<\infty .
$$

Note that

$$
1 \geq \frac{\tilde{r}_{j}}{\beta_{j}}=1-\frac{h_{1}}{\tilde{\kappa}^{j} \alpha_{0}} \quad \text { and } \quad 1 \leq \frac{\tilde{s}_{j}}{\beta_{j}}=\frac{\beta_{j}+h_{2}}{\beta_{j}+\delta-a}=1+\frac{h_{2}+a-\delta}{\tilde{\kappa}^{j} \alpha_{0}+\delta-a} .
$$

Then it is elementary to show that the products

$$
\tilde{\mu}=\prod_{j=0}^{\infty} \frac{\tilde{r}_{j}}{\beta_{j}} \quad \text { and } \quad \tilde{\nu}=\prod_{j=0}^{\infty} \frac{\tilde{s}_{j}}{\beta_{j}}
$$

are positive numbers. By (4.44) and Lemma 4.3, we obtain

$$
\limsup _{j \rightarrow \infty} Y_{j} \leq\left(2 A_{T, \sigma, \alpha_{0}}\right)^{\omega} \max \left\{Y_{0}^{\tilde{\mu}}, Y_{0}^{\tilde{\nu}}\right\}
$$

where $\omega=\mathcal{G} \sum_{j=0}^{\infty} \frac{j+1}{\beta_{j}}$ with $\mathcal{G}=\prod_{k=1}^{\infty}\left(\tilde{s}_{k} / \beta_{k}\right) \in(0, \infty)$.

Note that $Y_{0}=\|u\|_{L^{\beta_{1}\left(\mathcal{Q}_{0}\right)}}$ and, by (4.43),

$$
\left(2 A_{T, \sigma, \varphi}\right)^{\omega} \leq C\left(1+\frac{1}{\sigma T}\right)^{\omega_{1}}(1+T)^{\omega_{2}} \mathcal{M}_{0}^{\omega_{3}},
$$

where $\omega_{1}=2 \omega, \omega_{2}=3 \omega$ and $\omega_{3}=2 \omega$. Then estimate (4.38) follows (4.47).

Remark 4.5. Estimate 4.38) can be rewritten as

$$
\|u\|_{L^{\infty}(U \times(\sigma T, T))} \leq C_{\sigma, T, f_{1}, f_{2}, \varphi_{1}, \varphi_{2}}\left(\|u\|_{L^{\tilde{\kappa} \alpha_{0}(U \times(0, T))}}^{\tilde{\tilde{a}}}+\|u\|_{L^{\tilde{\kappa} \alpha_{0}(U \times(0, T))}}^{\tilde{\tilde{N}}}\right) .
$$

The bound on the right-hand side is quasi-homogeneous in $\|u\|_{L^{\tilde{\kappa} \alpha_{0}(U \times(0, T))}}$, hence, relevant for both small and large values of $\|u\|_{L^{\tilde{\kappa} \alpha_{0}(U \times(0, T))}}$. This is different from the commonly obtained estimates when a positive constant is added to the inequality's right-hand side. This global (in space) estimate extends our own version in [4], and the local one in [29].

Combining Theorems 3.2 and 4.4 gives the following particular estimates of in terms of initial and boundary data. 
Theorem 4.6. Let $\alpha_{0}$ satisfy

$$
\alpha_{0}>\max \left\{2, \alpha_{*}, \eta_{1}, \frac{\eta_{0}}{\tilde{\kappa}}\right\} \quad \text { and } \quad \alpha_{0} \geq \max \left\{\frac{n a}{(1-a) \tilde{\kappa}}, \frac{\eta_{2}}{\tilde{\kappa}-\kappa_{*}}, \frac{a-\delta}{\kappa_{f}-\tilde{\kappa}^{2}}\right\} .
$$

Let $\tilde{\mu}, \tilde{\nu}, \omega_{1}, \omega_{2}, \omega_{3}$ be the same constants as in Theorem 4.4 and denote $\beta_{1}=\tilde{\kappa} \alpha_{0}$.

(i) If $T>0$ satisfies (3.38) for $\alpha=\beta_{1}$, then for $0<\varepsilon<\min \{1, T\}$, one has

$$
\|u\|_{L^{\infty}(U \times(\varepsilon, T))} \leq C \varepsilon^{-\omega_{1}}(1+T)^{\omega_{2}} \mathcal{M}_{0}^{\omega_{3}} \cdot \max \left\{\left(\int_{0}^{T} \mathcal{V}(t) d t\right)^{\frac{\tilde{\mu}}{\beta_{1}}},\left(\int_{0}^{T} \mathcal{V}(t) d t\right)^{\frac{\tilde{\nu}}{\beta_{1}}}\right\},
$$

where $\mathcal{M}_{0}$ is defined by (4.4), and for $0 \leq t \leq T$

$$
\mathcal{V}(t)=\left\{\left(1+\int_{U} u_{0}(x)^{\beta_{1}} d x\right)^{-\nu_{4}\left(\beta_{1}\right)}-\frac{1}{C_{1}\left(\beta_{1}\right)} \int_{0}^{t} \Upsilon(\tau) d \tau\right\}^{-\frac{1}{\nu_{4}\left(\beta_{1}\right)}}
$$

(ii) If $T>0$ satisfies (3.40) for $\alpha=\beta_{1}$, then for $0<\varepsilon<\min \{1, T\}$, one has

$$
\|u\|_{L^{\infty}(U \times(\varepsilon, T))} \leq C \varepsilon^{-\omega_{1}}(1+T)^{\omega_{2}+\frac{\tilde{\nu}}{\beta_{1}}}\left(1+\left\|u_{0}(x)\right\|_{L^{\beta_{1}}(U)}\right)^{\tilde{\nu}} \mathcal{M}_{0}^{\omega_{3}} .
$$

Above $C$ is a positive constant independent of $T$ and $\varepsilon$.

Proof. Note that $\alpha_{0}$ satisfies (4.37), and $\alpha=\beta_{1}$ satisfies (3.10). The constant $C>0$ in this proof is generic.

(i) Applying estimate (4.38) to $\sigma T=\varepsilon$ we have

$$
\begin{aligned}
\|u\|_{L^{\infty}(U \times(\varepsilon, T)) \leq} & C \varepsilon^{-\omega_{1}}(1+T)^{\omega_{2}} \mathcal{M}_{0}^{\omega_{3}} \\
& \cdot \max \left\{\left(\int_{0}^{T} \int_{U}|u|^{\beta_{1}} d x d t\right)^{\frac{\tilde{\mu}}{\beta_{1}}},\left(\int_{0}^{T} \int_{U}|u|^{\beta_{1}} d x d t\right)^{\frac{\tilde{\nu}}{\beta_{1}}}\right\} .
\end{aligned}
$$

By (3.39), we have

$$
\int_{U} u^{\beta_{1}}(x, t) d x \leq \mathcal{V}(t)
$$

Then (4.52) and (4.53) yield (4.49).

(ii) If $T$ satisfies (3.40) with $\alpha=\beta_{1}$, then by (3.41), we have

$$
\int_{U} u^{\beta_{1}}(x, t) d x \leq 2\left(1+\int_{U} u_{0}^{\beta_{1}}(x) d x\right) \quad \text { for all } t \in[0, T] .
$$

Combining (4.52) with (4.54), and noticing that $\tilde{\mu}<\tilde{\nu}$, we obtain

$$
\begin{aligned}
& \|u\|_{L^{\infty}(U \times(\varepsilon, T)) \leq C \varepsilon^{-\omega_{1}}(1+T)^{\omega_{2}} \mathcal{M}_{0}^{\omega_{3}}} \\
& \quad \cdot \max \left\{\left(2 \int_{0}^{T}\left(1+\int_{U} u_{0}^{\beta_{1}}(x) d x\right) d t\right)^{\frac{\tilde{\mu}}{\beta_{1}}},\left(2 \int_{0}^{T}\left(1+\int_{U} u_{0}^{\beta_{1}}(x) d x\right) d t\right)^{\frac{\tilde{\nu}}{\beta_{1}}}\right\} \\
& \leq C \varepsilon^{-\omega_{1}}(1+T)^{\omega_{2}} \mathcal{M}_{0}^{\omega_{3}} \cdot(1+T)^{\frac{\tilde{\nu}}{\beta_{1}}}\left(1+\int_{U} u_{0}^{\beta_{1}}(x) d x\right)^{\frac{\tilde{\nu}}{\beta_{1}}},
\end{aligned}
$$

and estimate (4.51) follows. 


\section{$5 \quad$ Gradient estimates}

In Theorem 3.2 we have an estimate for $\int_{0}^{T} \int_{U}|\nabla u(x, t)|^{2-a} u(x, t)^{\alpha-\lambda-1} d x d t$. Note that the double integral is in both spatial and time variables, and it is not yet a direct estimate for the gradient. Therefore, we focus, in the following, on estimating $\int_{U}|\nabla u(x, t)|^{2-a} d x$ directly for $t>0$.

In connection with properties (1.26) and (1.27) in Assumption (A1), it is natural to make the following assumptions.

Assumption (A2). The function $Z(u)$ satisfies

$$
\left|Z^{\prime}(u)\right| \leq d_{4} u^{\ell_{Z}-1} \quad \forall u \in[0, \infty),
$$

for some constant $d_{4}>0$, and there are non-negative functions $\varphi_{3}(x, t)$ and $\varphi_{4}(x, t)$ defined on $\Gamma \times(0, \infty)$ such that

$$
\left|\frac{\partial B(x, t, u)}{\partial t}\right| \leq \varphi_{3}(x, t)+\varphi_{4}(x, t) u^{\ell_{B}} .
$$

We also assume a slightly stronger version of (1.27) and (1.28), namely,

$$
\begin{gathered}
|B(x, t, u)| \leq \varphi_{1}(x, t)+\varphi_{2}(x, t) u^{\ell_{B}} \\
|f(x, t, u)| \leq f_{1}(x, t)+f_{2}(x, t) u^{\ell_{f}}
\end{gathered}
$$

To deal with the boundary condition, we define for any $x \in \Gamma, t \geq 0, u \geq 0$

$$
Q(x, t, u)=\int_{0}^{u} B(x, t, v) d v .
$$

Then by Assumptions (A1) and (A2), we have

$$
|Q(x, t, u)| \leq \varphi_{1}(x, t) u+\varphi_{2}(x, t) u^{\ell_{B}+1}
$$

and

$$
\left|\frac{\partial Q(x, t, u)}{\partial t}\right| \leq \varphi_{3}(x, t) u+C \varphi_{4}(x, t) u^{\ell_{B}+1}
$$

\section{Assumption (A3).}

$$
2 \ell_{Z}>\lambda+1
$$

For our original problem (1.23), $\ell_{Z}=2 \lambda$, then condition (5.8) becomes $\lambda>1 / 3$. For slightly compressible fluids, $\lambda=1$. For ideal gases, $\lambda=1 / 2$. For isentropic gas flows, from the data in [34] or section III of [6], all values of the specific heat ratio $\gamma$ belong to the interval $(1,2)$, therefore $\lambda=1 /(1+\gamma)$, see (1.20), satisfies $1 / 3<\lambda<1 / 2$. Thus (5.8) is naturally met in all cases.

For the gradient estimates, same as in [2,11, we will make use of the following function

$$
H(\xi)=\int_{0}^{\xi^{2}} K(\sqrt{s}) d s \quad \text { for } \xi \geq 0 .
$$

The function $H(\xi)$ satisfies

$$
K(\xi) \xi^{2} \leq H(\xi) \leq 2 K(\xi) \xi^{2}
$$


and hence, as a consequence of (3.3) and (5.9),

$$
d_{3}\left(\xi^{2-a}-1\right) \leq H(\xi) \leq 2 d_{2} \xi^{2-a} .
$$

Based on the structure of the PDE in (1.25), we define an intermediate quantity

$$
\mathcal{I}(t)=\int_{U} H(|\nabla u(x, t)+Z(u(x, t))|) d x \quad \text { for } t \geq 0
$$

and for initial values

$$
\mathcal{Z}_{0}=\int_{U} u_{0}^{\lambda+1}(x) d x+\mathcal{I}(0)+\int_{\Gamma}\left(\varphi_{1}(x, 0) u_{0}(x)+\varphi_{2}(x, 0) u_{0}^{\ell_{B}+1}(x)\right) d \sigma .
$$

First, we estimate $\mathcal{I}(t)$ in terms of certain Lebesgue norms of $u(x, t)$.

Proposition 5.1. For $t>0$,

$$
\mathcal{I}(t) \leq 2 \mathcal{Z}_{0}+C\left\{t+1+\int_{U} u^{\eta_{3}}(x, t) d x+\int_{0}^{t} \int_{U} u^{\eta_{4}}(x, \tau) d x d \tau+N_{1}(t)+\int_{0}^{t} N_{2}(\tau) d \tau\right\}
$$

where $C$ is a positive constant,

$$
\begin{aligned}
& \eta_{3}=\max \left\{\frac{(2-a)\left(2 \ell_{B}+1\right)}{1-a},(2-a) \ell_{Z}\right\} \\
& \eta_{4}=\max \left\{\frac{(2-a)\left(2 \ell_{B}+1\right)}{1-a}, \frac{2 \ell_{Z}(2-a)}{a}, 2\left(2 \ell_{f}+1\right)\right\}
\end{aligned}
$$

and

$$
\begin{aligned}
& N_{1}(t)=\int_{\Gamma}\left(\varphi_{1}^{\eta_{5}}(x, t)+\varphi_{2}^{2}(x, t)\right) d \sigma \\
& N_{2}(t)=N_{1}(t)+\int_{\Gamma}\left(\varphi_{3}^{\eta_{5}}(x, t)+\varphi_{4}^{2}(x, t)\right) d \sigma+\int_{U}\left(f_{1}^{\eta_{6}}(x, t)+f_{2}^{4}(x, t)\right) d x
\end{aligned}
$$

with

$$
\eta_{5}=\frac{2\left(1+\ell_{B}\right)}{1+2 \ell_{B}}, \quad \eta_{6}=\max \left\{\frac{2\left(1+\ell_{f}\right)}{1+2 \ell_{f}}, \frac{4\left(2 \ell_{f}+1-\lambda\right)}{4 \ell_{f}+1-\lambda}\right\} .
$$

Proof. The proof is divided into three steps below. As usual, constants $C, C_{\varepsilon}$ are positive and generic.

Step 1. Multiplying the PDE in (1.25) by $u$, integrating over $U$ and using integration by parts, we have

$$
\begin{aligned}
\frac{\lambda}{\lambda+1} \frac{d}{d t} \int_{U} u^{\lambda+1} d x= & \int_{U} \nabla \cdot(K(|\nabla u+Z(u)|)(\nabla u+Z(u))) u d x+\int_{U} f u d x \\
= & -\int_{U} K(|\nabla u+Z(u)|)(\nabla u+Z(u)) \cdot \nabla u d x+\int_{\Gamma} B u d \sigma+\int_{U} f u d x \\
= & -\int_{U} K(|\nabla u+Z(u)|)|\nabla u+Z(u)|^{2} d x \\
& +\int_{U} K(|\nabla u+Z(u)|)(\nabla u+Z(u)) \cdot Z(u)+\int_{\Gamma} B u d \sigma+\int_{U} f u d x .
\end{aligned}
$$


On the right-hand side of the last equation, using relation (5.9) for the first integral, relations (3.2), (1.26) for the second integral, (1.27) for the third, and (1.28) for the last, we have

$$
\begin{aligned}
\frac{\lambda}{\lambda+1} \frac{d}{d t} \int_{U} u^{\lambda+1} d x \leq & -\frac{1}{2} \int_{U} H(|\nabla u+Z(u)|) d x+C \int_{U}|\nabla u+Z(u)|^{1-a} u^{\ell_{Z}} d x \\
& +\int_{\Gamma}\left(\varphi_{1}+\varphi_{2} u^{\ell_{B}}\right) u d \sigma+\int_{U}\left(f_{1}+f_{2} u^{\ell_{f}}\right) u d x \\
& \stackrel{\text { def }}{=}-\frac{1}{2} \mathcal{I}+J_{1}+J_{2}+J_{3} .
\end{aligned}
$$

In the following, $\varepsilon \in(0,1)$.

- For $J_{1}$, using Young's inequality with powers $\frac{2-a}{1-a}$ and $2-a$, and then relation (5.10),

$$
J_{1} \leq \varepsilon \mathcal{I}+C_{\varepsilon}+C_{\varepsilon} \int_{U} u^{\ell_{Z}(2-a)} d x .
$$

- For $J_{2}$, using Young's inequality, we have

$$
J_{2} \leq 2\left\|u^{1+\ell_{B}}\right\|_{L^{2}(\Gamma)}^{2}+N_{1}(t) .
$$

Next, applying inequality (2.6) for $\alpha=s=2\left(\ell_{B}+1\right), p=2-a$ to the boundary integral $\left\|u^{1+\ell_{B}}\right\|_{L^{2}(\Gamma)}^{2}$, we have

$$
J_{2} \leq \varepsilon \int_{U}|\nabla u|^{2-a} d x+C\|u\|_{L^{2\left(\ell_{B}+1\right)}}^{2\left(\ell_{B}+1\right)}+C_{\varepsilon}\|u\|_{L^{s_{1}}}^{s_{1}}+N_{1}(t)
$$

with

$$
s_{1}=2\left(\ell_{B}+1\right)+\frac{2 \ell_{B}+a}{1-a}=\frac{(2-a)\left(2 \ell_{B}+1\right)}{1-a} .
$$

By (2.2) and then relations (1.26), (5.10),

$$
\begin{aligned}
\int_{U}|\nabla u|^{2-a} d x & \leq 2 \int_{U}|\nabla u+Z(u)|^{2-a} d x+2 \int_{U}|Z(u)|^{2-a} d x \\
& \leq \frac{2}{d_{3}} \mathcal{I}+C+C \int_{U} u^{(2-a) \ell_{Z}} d x
\end{aligned}
$$

Thus

$$
J_{2} \leq \frac{2 \varepsilon}{d_{3}} \mathcal{I}+C+C \int_{U} u^{(2-a) \ell_{Z}} d x+C \int_{U} u^{2\left(\ell_{B}+1\right)}+C_{\varepsilon} \int_{U} u^{s_{1}} d x+N_{1}(t) .
$$

Since $(2-a) \ell_{Z}, 2\left(\ell_{B}+1\right), s_{1} \leq \eta_{3}$, by using Young's inequality, we have

$$
J_{2} \leq \frac{2 \varepsilon}{d_{3}} \mathcal{I}+C_{\varepsilon}+C_{\varepsilon} \int_{U} u^{\eta_{3}} d x+N_{1}(t)
$$

- For $J_{3}$, using Young's inequality

$$
J_{3} \leq 2 \int_{U} u^{2\left(\ell_{f}+1\right)} d x+\int_{U}\left(f_{1}^{\frac{2\left(1+\ell_{f}\right)}{1+2 \ell_{f}}}+f_{2}^{4}\right) d x .
$$

Combining (5.12), (5.13), (5.14) and (5.15) gives

$$
\begin{aligned}
& \frac{\lambda}{\lambda+1} \frac{d}{d t} \int_{U} u^{\lambda+1} d x+\left(\frac{1}{2}-\varepsilon-\frac{2 \varepsilon}{d_{3}}\right) \mathcal{I} \\
& \leq C_{\varepsilon}\left(1+\int_{U}\left(u^{\ell_{Z}(2-a)}+u^{\eta_{3}}+u^{2\left(\ell_{f}+1\right)}\right) d x\right)+N_{3}(t),
\end{aligned}
$$


where

$$
N_{3}(t)=N_{1}(t)+\int_{U}\left(f_{1}^{\frac{2\left(1+\ell_{f}\right)}{1+2 \ell_{f}}}(x, t)+f_{2}^{4}(x, t)\right) d x .
$$

Taking $\varepsilon$ sufficiently small and using Young's inequality show that

$$
\frac{d}{d t} \int_{U} u^{\lambda+1} d x+\mathcal{I} \leq C+C \int_{U} u^{s_{2}} d x+C N_{3}(t)
$$

where

$$
s_{2}=\max \left\{2\left(\ell_{f}+1\right), \eta_{3}\right\} .
$$

Step 2. Multiplying both sides of the PDE in (1.25) by $u_{t}$, integrating over $U$ and using the boundary condition, one has

$$
\lambda \int_{U} u^{\lambda-1} u_{t}^{2} d x+\int_{U} K(|\nabla u+Z(u)|)(\nabla u+Z(u)) \cdot \nabla u_{t} d x=\int_{\Gamma} B u_{t} d \sigma+\int_{U} f u_{t} d x .
$$

Then by (3.2), we have

$$
\begin{aligned}
& \lambda \int_{U} u^{\lambda-1} u_{t}^{2} d x+\int_{U} K(|\nabla u+Z(u)|)(\nabla u+Z(u)) \cdot(\nabla u+Z(u))_{t} d x \\
& =\int_{U} K(|\nabla u+Z(u)|)(\nabla u+Z(u)) \cdot(Z(u))_{t} d x+\int_{\Gamma} B u_{t} d \sigma+\int_{U} f u_{t} d x \\
& \leq C \int_{U}|\nabla u+Z(u)|^{1-a}\left|Z^{\prime}(u)\right|\left|u_{t}\right| d x+\int_{\Gamma} B u_{t} d \sigma+\int_{U} f u_{t} d x .
\end{aligned}
$$

Hence by definition of $H(\xi)$, we have

$$
\begin{aligned}
& \lambda \int_{U} u^{\lambda-1} u_{t}^{2} d x+\frac{1}{2} \frac{d}{d t} \int_{U} H(|\nabla u+Z(u)|) d x \\
& \leq C \int_{U}|\nabla u+Z(u)|^{1-a}\left|Z^{\prime}(u)\right|\left|u_{t}\right| d x+\int_{\Gamma} B u_{t} d \sigma+\int_{U} f u_{t} d x .
\end{aligned}
$$

- We bound the first term of the right hand side in (5.17) by (5.1) and then Young's inequality,

$$
\begin{aligned}
& C \int_{U}|\nabla u+Z(u)|^{1-a}\left|Z^{\prime}(u)\right|\left|u_{t}\right| d x \leq C \int_{U}|\nabla u+Z(u)|^{1-a} u^{\ell_{Z}-1}\left|u_{t}\right| d x \\
& \leq \varepsilon \int_{U}|\nabla u+Z(u)|^{2-a} d x+C_{\varepsilon} \int_{U} u^{(2-a)\left(\ell_{Z}-1\right)}\left|u_{t}\right|^{2-a} d x .
\end{aligned}
$$

By (5.10) and then using Young's inequality one more time on the last term, we have

$$
C \int_{U}|\nabla u+Z(u)|^{1-a}\left|Z^{\prime}(u)\right|\left|u_{t}\right| d x \leq \frac{\varepsilon}{d_{3}} \mathcal{I}+C+\frac{\lambda}{4} \int_{U} u^{\lambda-1} u_{t}^{2} d x+C_{\varepsilon} \int_{U} u^{\frac{(2-a)\left(2 \ell_{Z}-\lambda-1\right)}{a}} d x .
$$

- For the middle term on the right-hand side of (5.17), using definition (5.5) we have

$$
B(x, t, u(x, t)) u_{t}(x, t)=\frac{d}{d t} Q(x, t, u(x, t))-\left.\frac{\partial Q}{\partial t}\right|_{(x, t, u(x, t))} .
$$

Thus (5.2) yields

$$
\begin{aligned}
\int_{\Gamma} B u_{t} d \sigma & =\frac{d}{d t} \int_{\Gamma} Q(x, t, u) d \sigma-\int_{\Gamma} \frac{\partial Q(x, t, u)}{\partial t} d \sigma \\
& \leq \frac{d}{d t} \int_{\Gamma} Q(x, t, u) d \sigma+C \int_{\Gamma}\left(\varphi_{3} u+\varphi_{4} u^{\ell_{B}+1}\right) d \sigma
\end{aligned}
$$


For the last term of (5.19), similar to estimate (5.14) we have

$$
C \int_{\Gamma}\left(\varphi_{3} u+\varphi_{4} u^{\ell_{B}+1}\right) d \sigma \leq \frac{2 \varepsilon}{d_{3}} \mathcal{I}+C_{\varepsilon}+C_{\varepsilon} \int_{U} u^{\eta_{3}} d x+C \int_{\Gamma}\left(\varphi_{3}^{\eta_{5}}+\varphi_{4}^{2}\right) d \sigma .
$$

- For the last term of (5.17), using Cauchy's inequality, (5.4) and Young's inequality, we obtain

$$
\begin{aligned}
\int_{U} f u_{t} d x & \leq \frac{\lambda}{4} \int_{U} u^{\lambda-1} u_{t}^{2} d x+C \int_{U} f^{2} u^{1-\lambda} d x \\
& \leq \frac{\lambda}{4} \int_{U} u^{\lambda-1} u_{t}^{2} d x+C \int_{U}\left(f_{1}^{2}+f_{2}^{2} u^{2 \ell_{f}}\right) u^{1-\lambda} d x \\
& \leq \frac{\lambda}{4} \int_{U} u^{\lambda-1} u_{t}^{2} d x+C \int_{U} u^{2\left(2 \ell_{f}+1-\lambda\right)} d x+C \int_{U}\left(f_{1}^{\frac{4\left(2 \ell_{f}+1-\lambda\right)}{4 \ell_{f}+1-\lambda}}+f_{2}^{4}\right) d x
\end{aligned}
$$

Then combining (5.17), (5.18), (5.19), (5.20), and (5.21), we obtain

$$
\begin{aligned}
& \frac{\lambda}{2} \int_{U} u^{\lambda-1} u_{t}^{2} d x+\frac{1}{2} \frac{d}{d t} \mathcal{I}-\frac{d}{d t} \int_{\Gamma} Q(x, t, u) d \sigma \\
& \leq \frac{3 \varepsilon}{d_{3}} \mathcal{I}+C_{\varepsilon}+C_{\varepsilon} \int_{U}\left(u^{\frac{(2-a)\left(2 \ell_{Z}-\lambda-1\right)}{a}}+u^{\eta_{3}}+u^{2\left(2 \ell_{f}+1-\lambda\right)}\right) d x+C N_{4}(t),
\end{aligned}
$$

where

$$
N_{4}(t)=\int_{\Gamma}\left(\varphi_{3}^{\eta_{5}}+\varphi_{4}^{2}\right) d \sigma+\int_{U}\left(f_{1}^{\frac{4\left(2 \ell_{f}+1-\lambda\right)}{4 \ell_{f}+1-\lambda}}+f_{2}^{4}\right) d x
$$

Step 3. Let $t \in(0, T)$. Note by assumption (5.8) that the power $(2-a)\left(2 \ell_{Z}-\lambda-1\right) / a$ in $(5.22)$ is positive. Summing (5.16), (5.22) with sufficiently small $\varepsilon$, and using Young's inequalities, we have

$$
\frac{d}{d t}\left(\int_{U} u^{\lambda+1} d x+\mathcal{I}-\int_{\Gamma} Q(x, t, u) d \sigma\right) \leq C+C \int_{U} u^{s_{3}} d x+C\left(N_{3}(t)+N_{4}(t)\right)
$$

with

$$
s_{3}=\max \left\{s_{2}, \frac{(2-a)\left(2 \ell_{Z}-\lambda-1\right)}{a}, 2\left(2 \ell_{f}+1-\lambda\right)\right\} .
$$

For simplicity, we bound $s_{3} \leq \eta_{4}$, and $u^{s_{3}} \leq 1+u^{\eta_{4}}$. Also, the powers $\frac{2\left(1+\ell_{f}\right)}{1+2 \ell_{f}}$ and $\frac{4\left(2 \ell_{f}+1-\lambda\right)}{4 \ell_{f}+1-\lambda}$ of $f_{1}$ in $N_{3}(t)$ and $N_{4}(t)$ are less than or equal to $\eta_{6}$, then by Young's inequality

$$
N_{3}(t)+N_{4}(t) \leq C\left(1+N_{2}(t)\right)
$$

Therefore, (5.24) yields

$$
\frac{d}{d t}\left(\int_{U} u^{\lambda+1} d x+\mathcal{I}-\int_{\Gamma} Q(x, t, u) d \sigma\right) \leq C+C \int_{U} u^{\eta_{4}} d x+C N_{2}(t)
$$

Integrating (5.25) in time from 0 to $t$ gives

$$
\begin{aligned}
\int_{U} u^{\lambda+1}(x, t) d x+\mathcal{I}(t) \leq \int_{U} u_{0}(x)^{\lambda+1} d x+\mathcal{I}(0) & -\int_{\Gamma} Q\left(x, 0, u_{0}(x)\right) d \sigma+\int_{\Gamma} Q(x, t, u) d \sigma \\
& +C t+C \int_{0}^{t} \int_{U} u^{\eta_{4}} d x d \tau+C \int_{0}^{t} N_{2}(\tau) d \tau
\end{aligned}
$$


We will neglect the first term on the left-hand side of (5.26). On the right-hand side, by (5.6) we have

$$
\int_{\Gamma}\left|Q\left(x, 0, u_{0}(x)\right)\right| d \sigma \leq \int_{\Gamma}\left(\varphi_{1}(x, 0) u_{0}(x)+\varphi_{2}(x, 0) u_{0}^{\ell_{B}+1}(x)\right) d \sigma
$$

while for $t>0$, using also estimate (5.14) we obtain

$$
\int_{\Gamma} Q(x, t, u) d \sigma \leq \int_{\Gamma}\left(\varphi_{1} u+\varphi_{2} u^{\ell_{B}+1}\right) d \sigma \leq \frac{2 \varepsilon}{d_{3}} \mathcal{I}+C_{\varepsilon}+C_{\varepsilon} \int_{U} u^{\eta_{3}} d x+C N_{1}(t) .
$$

Again, choosing $\varepsilon$ sufficiently small, we derive from (5.26) and (5.27) that

$$
\frac{1}{2} \mathcal{I}(t) \leq \mathcal{Z}_{0}+C(t+1)+C \int_{0}^{t} \int_{U} u^{\eta_{4}} d x d \tau+C \int_{0}^{t} N_{2}(\tau) d \tau+C \int_{U} u^{\eta_{3}} d x+C N_{1}(t),
$$

and, hence, estimate (5.11) follows.

Finally, we combine the estimate in Proposition 5.1 with those in Theorem 3.2 .

Theorem 5.2. Let

$$
\eta_{7}=\max \left\{\frac{n a}{1-a}, \eta_{0}+1, \eta_{4}\right\} \quad \text { and } \quad \eta_{8}=\nu_{4}\left(\eta_{7}\right)
$$

(i) If $T>0$ satisfies (3.38) for $\alpha=\eta_{7}$, then for all $t \in(0, T]$

$$
\int_{U}|\nabla u(x, t)|^{2-a} d x \leq C\left(\mathcal{Z}_{0}+(t+1)\left(1+\mathcal{V}_{*}(t)\right)+N_{1}(t)+\int_{0}^{t} N_{2}(\tau) d \tau\right),
$$

where

$$
\mathcal{V}_{*}(t)=\left\{\left(1+\int_{U} u_{0}(x)^{\eta_{7}} d x\right)^{-\eta_{8}}-\frac{1}{C_{1}\left(\eta_{7}\right)} \int_{0}^{t}(1+\Upsilon(\tau)) d \tau\right\}^{-\frac{1}{\eta_{8}}}
$$

(ii) Moreover, if $T>0$ satisfies (3.40) for $\alpha=\eta_{7}$ then for all $t \in(0, T]$

$$
\int_{U}|\nabla u(x, t)|^{2-a} d x \leq C\left\{\mathcal{Z}_{0}+(t+1)\left(1+\int_{U} u_{0}^{\eta_{7}}(x) d x\right)+N_{1}(t)+\int_{0}^{t} N_{2}(\tau) d \tau\right\} .
$$

Above, $C$ is a positive constant.

Proof. Thanks to (2.2), (5.10) and (5.11), we have

$$
\begin{aligned}
& \int_{U}|\nabla u|^{2-a} d x \leq 2 \int_{U}\left(|\nabla u+Z(u)|^{2-a}+|Z(u)|^{2-a}\right) d x \leq C \mathcal{I}(t)+C+C \int_{U} u^{\ell_{Z}(2-a)} d x \\
& \leq C\left\{\mathcal{Z}_{0}+(t+1)+\int_{U}\left(u^{\eta_{3}}+u^{\ell_{Z}(2-a)}\right) d x+\int_{0}^{t} \int_{U} u(x, \tau)^{\eta_{4}} d x d \tau+N_{1}(t)+\int_{0}^{t} N_{2}(\tau) d \tau\right\} .
\end{aligned}
$$

Using the fact $\eta_{3}, \ell_{Z}(2-a), \eta_{4} \leq \eta_{7}$ and (2.4) to bound $u^{\eta_{3}}, u^{\ell_{Z}(2-a)}, u^{\eta_{4}} \leq 1+u^{\eta_{7}}$, we obtain

$$
\begin{aligned}
\int_{U}|\nabla u|^{2-a} d x \leq & C\left\{\mathcal{Z}_{0}+(t+1)+\int_{U} u(x, t)^{\eta_{7}} d x+\int_{0}^{t} \int_{U} u(x, \tau)^{\eta_{7}} d x d \tau\right. \\
& \left.+N_{1}(t)+\int_{0}^{t} N_{2}(\tau) d \tau\right\} .
\end{aligned}
$$


(i) Note that $\alpha=\eta_{7}$ satisfies (3.10). Let $T>0$ satisfy (3.38) for $\alpha=\eta_{7}$. Now using (3.39) with $\alpha=\eta_{7}$, we have

$$
\int_{U} u(x, t)^{\eta_{7}} d x \leq \mathcal{V}_{*}(t)
$$

By (5.31) and the fact $\mathcal{V}_{*}(t)$ is increasing in $t$, we have

$$
\int_{U}|\nabla u|^{2-a} d x \leq C\left\{\mathcal{Z}_{0}+(t+1)+(t+1) \mathcal{V}_{*}(t)+N_{1}(t)+\int_{0}^{t} N_{2}(\tau) d \tau\right\},
$$

and, therefore, obtain (5.28).

(ii) Now, assume $T>0$ satisfies (3.38) for $\alpha=\eta_{7}$. By using (3.41) instead of (3.39), we can replace $\mathcal{V}_{*}(t)$ with $2\left(1+\int_{U} u_{0}^{\eta_{7}}(x) d x\right)$ in (5.31) and (5.32), and consequently, estimate (5.29) follows.

Acknowledgement. L. H. acknowledges the support by NSF grant DMS-1412796.

\section{References}

[1] Alt, H. W., And Luckhaus, S. Quasilinear elliptic-parabolic differential equations. Math. Z. 183,3 (1983), 311-341.

[2] Aulisa, E., Bloshanskaya, L., Hoang, L., and Ibragimov, A. Analysis of generalized Forchheimer flows of compressible fluids in porous media. J. Math. Phys. 50, 10 (2009), 103102:44pp.

[3] Bear, J. Dynamics of Fluids in Porous Media. American Elsevier Pub. Co., New York, 1972.

[4] Celik, E., Hoang, L., and Kieu, T. Generalized Forchheimer flows of isentropic gases. submitted, preprint http://arxiv.org/abs/1504.00742.

[5] Darcy, H. Les Fontaines Publiques de la Ville de Dijon. Dalmont, Paris, 1856.

[6] de Lange, O. L., And Pierrus, J. Measurement of bulk moduli and ratio of specific heats of gases using Rüchardts experiment. Am. J. Phys. 68, 3 (2000), 265-270.

[7] Diaz, J. I., AND DE ThÉLin, F. On a nonlinear parabolic problem arising in some models related to turbulent flows. SIAM J. Math. Anal. 25, 4 (1994), 1085-1111.

[8] Douglas, J. J., Paes-Leme, P. J., And Giorgi, T. Generalized Forchheimer flow in porous media. In Boundary value problems for partial differential equations and applications, vol. 29 of RMA Res. Notes Appl. Math. Masson, Paris, 1993, pp. 99-111.

[9] Forchheimer, P. Wasserbewegung durch Boden. Zeit. Ver. Deut. Ing. 45 (1901), 1781-1788.

[10] Forchheimer, P. Hydraulik. No. Leipzig, Berlin, B. G. Teubner. 1930. 3rd edition.

[11] Hoang, L., And Ibragimov, A. Structural stability of generalized Forchheimer equations for compressible fluids in porous media. Nonlinearity 24, 1 (2011), 1-41.

[12] Hoang, L., And Ibragimov, A. Qualitative study of seneralized Forchheimer flows with the flux boundary condition. Adv. Diff. Eq. 17, 5-6 (2012), 511-556. 
[13] Hoang, L., Ibragimov, A., Kieu, T., And Sobol, Z. Stability of solutions to generalized Forchheimer equations of any degree. J. Math. Sci. 210, 4 (2015), 476-544.

[14] HoAng, L., AND Kieu, T. Interior estimates for generalized Forchheimer flows of slightly compressible fluids. submitted, preprint http://arxiv.org/abs/1404.6517.

[15] Hoang, L., And Kieu, T. Global estimates for generalized Forchheimer flows of slightly compressible fluids. Journal d'Analyse Mathematique (2015). accepted.

[16] Hoang, L. T., Ibragimov, A., and Kieu, T. T. One-dimensional two-phase generalized Forchheimer flows of incompressible fluids. J. Math. Anal. Appl. 401, 2 (2013), 921-938.

[17] Hoang, L. T., Ibragimov, A., and Kieu, T. T. A family of steady two-phase generalized Forchheimer flows and their linear stability analysis. J. Math. Phys. 55, 12 (2014), 123101:32pp.

[18] Hoang, L. T., Kieu, T. T., And Phan, T. V. Properties of generalized Forchheimer flows in porous media. J. Math. Sci. 202, 2 (2014), 259-332.

[19] Ivanov, A. V. Second-order quasilinear degenerate and nonuniformly elliptic and parabolic equations. Trudy Mat. Inst. Steklov. 160 (1982), 285.

[20] Ivanov, A. V. The regularity theory for $(M, L)$-Laplacian parabolic equation. Zap. Nauchn. Sem. S.-Peterburg. Otdel. Mat. Inst. Steklov. (POMI) 243, Kraev. Zadachi Mat. Fiz. i Smezh. Vopr. Teor. Funktsii. 28 (1997), 87-110, 339.

[21] KIEU, T. Analysis of expanded mixed finite element methods for the generalized Forchheimer flows of slightly compressible fluids. Numer. Methods Partial Differential Equations (2015). accepted.

[22] Kinnunen, J., And KuUsi, T. Local behaviour of solutions to doubly nonlinear parabolic equations. Mathematische Annalen 337, 3 (2007), 705-728.

[23] Manfredi, J. J., And Vespri, V. Large time behavior of solutions to a class of doubly nonlinear parabolic equations. Electron. J. Differential Equations (1994), No. 02, approx. 17 pp. (electronic only).

[24] Moser, J. On a pointwise estimate for parabolic differential equations. Communications on Pure and Applied Mathematics 24, 5 (1971), 727-740.

[25] Muskat, M. The flow of homogeneous fluids through porous media. McGraw-Hill Book Company, inc., 1937.

[26] Nield, D. A., And Bejan, A. Convection in porous media, fourth ed. Springer-Verlag, New York, 2013.

[27] PARK, E.-J. Mixed finite element methods for generalized Forchheimer flow in porous media. Numer. Methods Partial Differential Equations 21, 2 (2005), 213-228.

[28] Straughan, B. Stability and wave motion in porous media, vol. 165 of Applied Mathematical Sciences. Springer, New York, 2008. 
[29] Surnachëv, M. D. On improved estimates for parabolic equations with double degeneration. Tr. Mat. Inst. Steklova 278, Differentsialnye Uravneniya i Dinamicheskie Sistemy (2012), 250259 .

[30] Tsutsumi, M. On solutions of some doubly nonlinear degenerate parabolic equations with absorption. J. Math. Anal. Appl. 132, 1 (1988), 187-212.

[31] VÁzQuez, J. L. The porous medium equation. Oxford Mathematical Monographs. The Clarendon Press Oxford University Press, Oxford, 2007. Mathematical theory.

[32] Vespri, V. On the local behaviour of solutions of a certain class of doubly nonlinear parabolic equations. Manuscripta Math. 75, 1 (1992), 65-80.

[33] Ward, J. C. Turbulent flow in porous media. Journal of the Hydraulics Division, Proc. Am. Soc. Civ. Eng. 90(HY5) (1964), 1-12.

[34] Yaws, C. L. Matheson gas data book, 7th ed. Matheson Tri-Gas, NJ, 2001. 